\title{
A Multi-Scale Model for Simulating Liquid-Fabric Interactions
}

\author{
YUN (RAYMOND) FEI, Columbia University, USA \\ CHRISTOPHER BATTY, University of Waterloo, Canada \\ EITAN GRINSPUN and CHANGXI ZHENG, Columbia University, USA
}

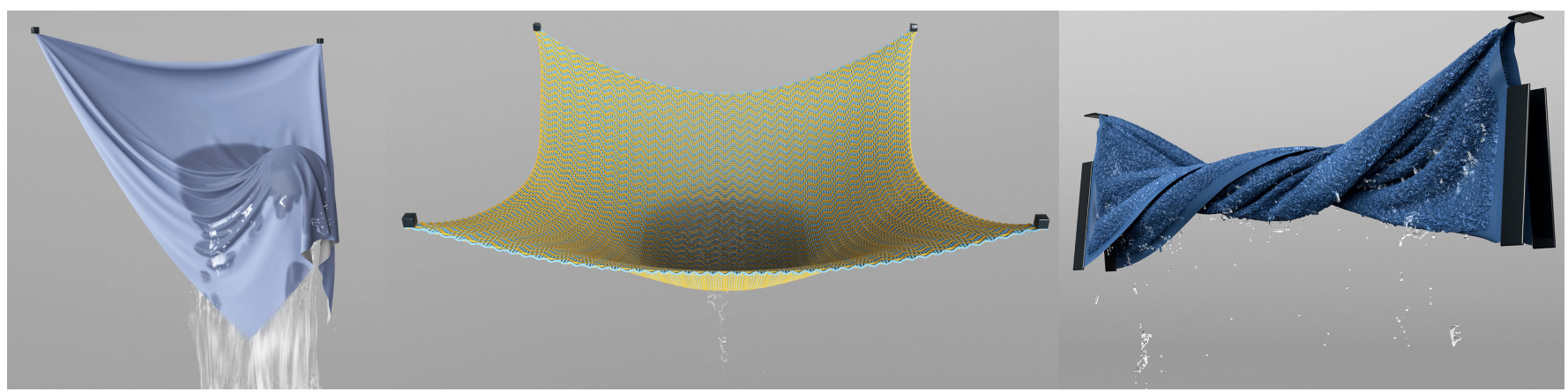

Fig. 1. Left: A piece of mesh-based cloth draped over a solid obstacle is splashed with water. Middle: Water flows through a piece of yarn-based handwoven fabric. Right: After being vigorously wrung out, a fuzzy towel continues to drip.

We propose a method for simulating the complex dynamics of partially and fully saturated woven and knit fabrics interacting with liquid, including the effects of buoyancy, nonlinear drag, pore (capillary) pressure, dripping, and convection-diffusion. Our model evolves the velocity fields of both the liquid and solid relying on mixture theory, as well as tracking a scalar saturation variable that affects the pore pressure forces in the fluid. We consider the porous microstructure implied by the fibers composing individual threads, and use it to derive homogenized drag and pore pressure models that faithfully reflect the anisotropy of fabrics. In addition to the bulk liquid and fabric motion, we derive a quasi-static flow model that accounts for liquid spreading within the fabric itself. Our implementation significantly extends standard numerical cloth and fluid models to support the diverse behaviors of wet fabric, and includes a numerical method tailored to cope with the challenging nonlinearities of the problem. We explore a range of fabricwater interactions to validate our model, including challenging animation scenarios involving splashing, wringing, and collisions with obstacles, along with qualitative comparisons against simple physical experiments.

CCS Concepts: • Computing methodologies $\rightarrow$ Physical simulation;

Additional Key Words and Phrases: wet cloth, wet yarn, fluid dynamics, two-way coupling, multi-scale

Authors' addresses: Yun (Raymond) Fei, Columbia University, Computer Science, New York, NY, 10027, USA; Christopher Batty, University of Waterloo, Computer Science, Waterloo, ON, N2L 3G1, Canada; Eitan Grinspun; Changxi Zheng, Columbia University, Computer Science, New York, NY, 10027, USA.

Permission to make digital or hard copies of all or part of this work for personal or classroom use is granted without fee provided that copies are not made or distributed for profit or commercial advantage and that copies bear this notice and the full citation on the first page. Copyrights for components of this work owned by others than ACM must be honored. Abstracting with credit is permitted. To copy otherwise, or republish, to post on servers or to redistribute to lists, requires prior specific permission and/or a fee. Request permissions from permissions@acm.org.

(C) 2018 Association for Computing Machinery.

0730-0301/2018/8-ART51 \$15.00

https://doi.org/10.1145/3197517.3201392

\section{ACM Reference Format:}

Yun (Raymond) Fei, Christopher Batty, Eitan Grinspun, and Changxi Zheng. 2018. A Multi-Scale Model for Simulating Liquid-Fabric Interactions. ACM Trans. Graph. 37, 4, Article 51 (August 2018), 16 pages. https://doi.org/10. $1145 / 3197517.3201392$

\section{INTRODUCTION}

A beach vacation provides ample opportunity to experience the characteristic aspects of liquid-fabric interaction. A tipped piña colada splashes onto beachwear, wetting, then diffusing to dampen a larger area. Submerged board shorts drag along with the ocean waves, lifted buoyantly upon the surf, then drip distinctively on the return to dry land. Wringing those shorts then squeezes out the liquid, leaving tiny drops scattered within the fabric microstructure.

To develop a computational model of these varied liquid-fabric interactions, we must understand the composition of fabric. Fabric is composed of individual strands ("thread," "yarn") packed into thin oriented fibers. Tiny pockets within and between these fibers collect fluid, and are largely responsible for the wetting behavior we observe at the coarse scale. Because these pockets are numerous and individually imperceptible to the naked eye, it can be wasteful or intractable to represent them as discrete elements for animation applications. Therefore, we develop a macroscopic model.

Building on modern mixture theory [Anderson and Jackson 1967], we model fabric as a continuous porous medium through which fluid may flow. The model accounts for the material's anisotropic structure, and the evolution of its saturation, to capture buoyancy, drag, small-scale capillary (surface tension) effects, and fluid convection.

Our numerical treatment integrates a piecewise linear Lagrangian cloth or rod model [Bergou et al. 2010; Grinspun et al. 2003] with a hybrid Eulerian-Lagrangian (APIC) fluid simulator [Bridson 2015; Jiang et al. 2015]. We apply this model to application scenarios involving mesh-based cloth, yarn-based fabric, and fuzzy fabric 
in contact with water. We also examine qualitative comparisons against simple real-world experiments, including liquid spreading and suction tests.

We develop a multi-scale framework capturing the interactions between fabric and fluid, including

- the adaptation of mixture theory and porous flow to partially saturated fabrics with buoyancy in a particle-in-cell framework,

- the development of an approximate anisotropic fabric microstructure model to support nonlinear drag and pore pressure forces,

- treatments for liquid capture, and dripping,

- a quasi-static model of fluid flow within the fabric based on convection-diffusion,

- an efficient numerical solver for the resulting complex systems.

\section{RELATED WORK}

Cloth Simulation. Cloth simulation has a long history in computer animation; we refer to the survey of Thomaszewski et al. [2007] for a thorough review. Two of the key aspects of a cloth simulation system are the numerical model for the cloth dynamics and the approach used for contact- and collision-handling. In our work, we adopt the discrete shell model [Grinspun et al. 2003] to treat the bending of cloth and linear elasticity [Bonet and Wood 1997] for stretching, based on their simplicity and effectiveness. To handle contacts and collisions, we make use of the recently proposed method of Jiang et al. [2017] which exploits a background volumetric grid to efficiently treat contact forces among complex colliding materials, drawing on ideas from material point methods (MPM) [Sulsky et al. 1994]. We adapt their method with augmented MPM [Stomakhin et al. 2014] to use the marker-and-cell (MAC) grid for pressure projection. Nevertheless, our approach is not intrinsically dependent on these choices, and should be compatible with other cloth simulation frameworks.

Yarn Simulation. Since real cloth is composed of many individual threads, a more costly but potentially much more faithful strategy is to simulate every strand of yarn or thread. This was first suggested by Kaldor et al. [2008], and further accelerated by Kaldor et al. [2010] and Cirio et al. [2014] with more efficient treatments of inter-yarn contact. As noted above, we instead make use of the work by Jiang et al. [2017] that models yarns as Lagrangian rods, but handles complicated collisions on a grid.

Liquid simulation. To treat bulk liquid regions, we adopt a hybrid $\mathrm{grid} /$ particle-based simulator that uses the affine particle-in-cell (APIC) method [Jiang et al. 2015]. A thorough review of hybrid and grid-based methods for fluid simulation is provided by Bridson [2015]. A common alternative that has also been frequently used for fluid-cloth interactions is the family of smoothed particle hydrodynamics (SPH) methods [Ihmsen et al. 2014; Monaghan 1994; Müller et al. 2003]. Our use of APIC allows our method to share the usual benefits of mixed Eulerian-Lagrangian approaches, including simplicity of boundary handling, efficacy of pressure computation, and ease of integration into visual effects pipelines.
Wetting of solids in animation. The earliest explorations of wetting effects addressed painting techniques or the simulation of flows on static planar objects. Curtis et al. [1997] simulated shallow water on paper textiles for watercolor painting effects, and solved a diffusion equation to treat capillary effects that enable spreading of fluid through pores in paper. Later, Chu and Tai [2005] proposed a sophisticated system to simulate the ink percolation process. In their work the permeability and boundary conditions are designed based on artistic considerations. Instead of solving a simple diffusion flow, Huber et al. [2011] solved Fick's second law on cloth with a gravitational term added, and also demonstrated liquid absorption.

Fluid-solid interaction is a many-faceted phenomenon, and some previous works have therefore sought to address one or two of those facets in isolation. With an approach relying on fractional derivatives, Ozgen et al. [2010] simulated the deformation of completely submerged cloth without simulating water at all. Chen et al. [2012] proposed modified saturation, wrinkling, and friction models to better approximate the look of wet clothing. Um et al. [2013] combined a shallow water model and the diffusion equation to address fluid flow on and within dynamic cloth.

Another branch of research has focused on careful handling of boundary conditions for water interacting with impermeable thin shells, for both Eulerian and Lagrangian fluids. In the context of Eulerian methods, Guendelman et al. [2005] used a variable-density pressure solve to account for weakly coupled interaction forces, while Robinson et al. [2008] proposed a strong coupling approach by temporarily lumping together the momentum of thin shells and fluid. Azevedo et al. [2016] used conforming interpolation and exact cut cells to prevent fluid crossing over impermeable thin boundaries. Among SPH methods, Akinci et al. [2013] carefully sampled thin deformable objects with SPH particles to improve the accuracy of pressure forces and ensure the cloth remains impermeable to liquid, assuming appropriate timestep sizes. Huber et al. [2015] instead used the cloth triangle mesh itself directly, combining repulsion forces and continuous collision detection to strictly enforce impermeability. In this paper we target permeable thin structures, and therefore take a weak coupling approach that uses drag and buoyancy forces to transfer momentum between liquid and thin structures.

Simulation of mixtures in animation. Simulation of more general deformable wet materials, including cloth, was proposed by Lenaerts et al. [2008]. They used an SPH method to solve porous (Darcy) flow inside a solid object. Similarly, Rungjiratananon et al. [2008] considered fluid interactions with dynamic porous media in the context of wet sand, simulating sand, water, and their mutual interactions using SPH. Subsequent research focused on various simplifications intended to achieve higher performance. Saket and Parag [2013] presented an SPH method for the simulation of wet cloth, using a geometric diffusion method to simulate interior flow for increased efficiency. Lin et al. [2014; 2015] proposed a similar porous flow model with SPH, but further incorporated two-way fluid-hair interactions. Fei et al. [2017] coupled an APIC-based water simulator with a dimension-reduced model for thin liquid on elastic rods to treat fluid-hair interactions, assuming the hairs to be impermeable.

Mixture theory was first introduced for animation by Nielsen and Østerby [2013], who simulated fluid spray and air as continua. 
Later, Ren et al. [2014] and Yang et al. [2015] proposed an SPH-based framework to handle a wide range of multi-fluid flow phenomena including extraction and partial dissolution. Yan et al. [2016] generalized the multi-fluid SPH framework to incorporate solids, adopting a diffusion model for the relative motion between solid and liquid. More recently, Yang et al. [2017] extended their previous SPH framework with a phase-field method to simulate phase-changing phenomena for multi-materials. In their method, the momentum exchange between different phases was done by incorporating a viscous term between particles, and inside each particle different materials share the same momentum. We adopted a similar physical model, but solved the equations on both polygonal meshes and an Eulerian grid, allowing us to capture the diffusion and pressure forces more accurately, and incorporate stiff elastoplastic materials with large drag forces more effectively.

In recent work on simulating porous sand mixed with water [Tampubolon et al. 2017], the authors adopted a formulation by Bandara and Soga [2015] to compute buoyancy forces, but concluded that buoyancy is largely negligible in their problem. In our setting, the buoyancy force significantly affects the motion, as demonstrated in Fig. 4 and the supplemental video. Furthermore, we adopt a nonlinear drag force that is appropriate for liquid at both low and high Reynolds numbers, and confirm with dimensional analysis that our drag force is physically consistent.

Modeling porous media. The history of modeling porous media can be traced back to the late 18th century, when empirical models for fluid and porous solids were adopted to solve hydraulics problems for architectural design [Woltmann 1792]. A review of works in the early era can be found in the survey by Bedford and Drumheller [1983] or the book by de Boer [2012]. Some physical models developed during this era are still widely used today in numerical simulations. For example, Fick's second law [1855] can be used to describe moisture transmission through homogeneous fabric material [Das et al. 2007]. Darcy's law [1856] can be used to calculate the velocity of liquid through porous media for a given pressure drop, viscosity coefficient, and permeability, and is a popular choice for the calculation of viscous drag in a numerical simulation [Bandara and Soga 2015].

Subsequent research sought to improve the accuracy of drag models. Forchheimer [1901] extended Darcy's (linear) drag model with a quadratic model for high Reynolds number flows. Ergun [1952] extended the empirical Kozeny-Carman equation [Carman 1937] (another extension of Darcy's law for modeling linear permeability) and proposed a non-linear version which is a function of the Reynolds number. The Ergun equation can also be reformulated to discover the relationship between linear and non-linear drag forces, which can be applied to various materials [Akgiray and Saatç1 2001; Nithiarasu et al. 1997]. In this paper, we adopt a modern, unified drag formulation [Yazdchi and Luding 2012], and use the Ergun equation to relate the linear and non-linear terms, while the permeability of fibers is calculated following the empirically determined equations of Stylianopoulos et al. [2008].

Early soil mechanics researchers studied the effect of water pressure on soil. Fillunger [1913] and Terzaghi [1923] found that the total stress applied on a mixture is the combination of the effective stress (compression and shear resistance) and the pore water pressure, an effect which is now known as Terzaghi's principle. Later Biot [1941] combined Terzaghi's principle with linear elasticity and fluid dynamics to develop the theory of dynamic poroelasticity (sometimes called Biot's theory), which became the foundation of mixture theory [Anderson and Jackson 1967]. Mixture theory was initially developed for saturated porous media with incompressible solids, where the interaction forces between porous solids and liquid include two parts: drag and pore pressure. Pore pressure is usually formulated as the pressure gradient applied to the solid and fluid with their respective volume fractions [Pitman and Le 2005]. Recently, Borja [2006] generalized mixture theory for unsaturated porous media with compressible solids, and formalized it in a mathematical framework [Song and Borja 2014]. Their formulation has been used in several papers simulating two-way coupled porous media. For example, Abe et al. [2013] used the material point method (MPM) to solve the generalized Darcy equation for the simulation of creeping flow in porous soil. Bandara and Soga [2015] later extended this method to include the inertial effects of liquid to address porous media undergoing large deformations. Daviet and Bertails-Descoubes [2017] combined mixture theory with an implicit non-smooth treatment of the Drucker-Prager rheology to simulate immersed granular flows.

Our method is also built on mixture theory, and was inspired by these methods from engineering. We derive a two-scale mixture model targeting bulk fluid and codimensional porous flow, respectively, to simulate thin, unsaturated porous media undergoing large deformations. For the flow inside cloth/yarn, instead of combining another model, we show that the codimensional flow is a specific case of the equations for bulk liquids and solids, and can be derived from mixture theory.

Reduced flows. Reduced models for fluid flows have been an active topic of research for centuries. For example, the original shallow water equations [de Saint-Venant 1856] describe flow on planar boundaries where the vertical velocity is negligible; Hele-Shaw flow [Hele-Shaw 1898] describes non-inertial flow between two thin plates; and lubrication theory [Oron et al. 1997] is used to model the dynamics of thin liquid whose viscosity dominates over inertia. A thorough introduction to such reduced flows can be found in the book by Ockendon [1995]. Within computer animation, Wang et al. [2007] generalized the shallow water equations to mesh surfaces. Segall et al. [2016] proposed an efficient model for Hele-Shaw flow using generalized barycentric coordinates. Azencot and Vantzos [2015; 2017] proposed a numerical scheme to efficiently evolve thin film flow on arbitrary meshes. Inspired by these previous works, we adopt similar discrete differential operators to discretize a generalized variant of the Richards equation [Richards 1931] on cloth, yarn, and junctions between them.

Elastocapillarity in Textiles. At small scales, surface tension forces on liquid-air interfaces exhibit elastocapillarity, in which liquid "bridges" arise that can deform elastic solids, as survey recently by Bico et al. [2018]. Although the cohesion between two planar objects has been extensively researched [Wang et al. 2013], this effect was not methodically studied on textiles until recent work by 


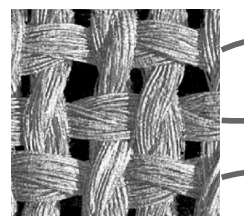

(a)

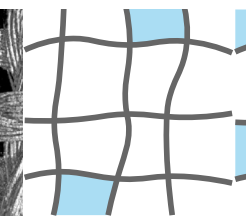

(b)

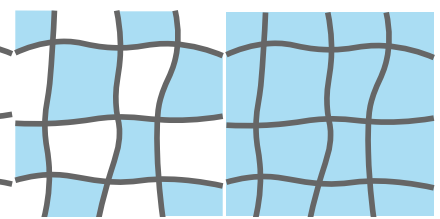

(c) (d)

Fig. 2. Fabric as porous material. (a) Micro-CT image of plain woven fabric, adapted from [Shinohara et al. 2010]. (b) Barely-saturated fabric $\left(S_{\mathrm{r}} \approx 0.1\right)$. (c) Half-saturated fabric $\left(S_{\mathrm{r}} \approx 0.5\right)$. (d) Fully-saturated fabric $\left(S_{\mathrm{r}}=1.0\right)$.

Lou et al. [2015; 2018; 2017]. The authors considered liquid bridges with circular area, and showed that the coalescence force between textile and water increases monotonically with the perimeter of the circular wetted area. Inspired by this work, we adopt a simple model to approximate the perimeter of the wetted area and calculate the corresponding cohesion force.

Wicking in Textiles. Research in textile engineering has studied how the pores in cloth and yarn affect the behavior of liquid propagation, or wicking [Kissa 1996]. Cloth and yarn are usually modeled as capillary tubes and the classic Lucas-Washburn equation [Lucas 1918; Washburn 1921] has been widely applied to the prediction of the position of the hydraulic head in one-dimensional scenarios. A detailed discussion on this topic can be found in the book by Masoodi and Pillai [2012b]. Modern research focuses on the experimental estimation of the capillary radius [Dang-Vu and Hupka 2005; Masoodi et al. 2008], which is the effective radius of pores, and on modeling the suction tensor that describes the stress due to surface tension [Scholtès et al. 2009]. Wicking along fibers was studied by Chwastiak [1973], Amico and Lekakou [2002], and Williams et al. [1974]; wicking across fibers was investigated by Senoguz et al. [2001], Ahn et al. [1991], Lekakou and Bader [1998], and Pillai and Advani [1996]. Further work has studied the suction tensor in woven or non-woven fabrics [Ahn et al. 1991; Kim 2003]. In our work, we adopt a general model for textiles from Masoodi and Pillai [2012a] and propose to construct the anisotropic suction tensor by aligning to specific axes. Several of our examples show the effect of wicking in cloth or yarn.

\section{PHYSICAL MODEL OF WET FABRIC}

We represent wet fabric as a continuum mixture of water, air, and fabric material (Figure 2). The governing equations for such a continuum are provided by mixture theory.

\subsection{Mixture Theory}

Mixture theory [Rajagopal and Tao 1995] models multiphase systems consisting of several interpenetrable continua. The theory assumes that all three phases are present, in some ratio, at every point of the material. The theory develops the momentum and mass balance equations for such a mixture.

As water penetrates it, fabric saturates from dry to damp to soaked (Figure 2). Saturation is the measure that determines volume fractions, the relative occupancy of the water, air, and solid fabric.

Saturated continuity equations. In the (maximally) saturated state, fabric pores are entirely filled with liquid [Anderson and Jackson 1967; Daviet and Bertails-Descoubes 2017]. The motion of both the porous medium and the liquid are described by

$$
\begin{aligned}
& \rho_{\mathrm{s}} \phi \frac{\mathrm{D}_{\boldsymbol{u}_{\mathrm{s}}} \boldsymbol{u}_{\mathrm{s}}}{\mathrm{D}_{\boldsymbol{u}_{\mathrm{s}}} t}-\nabla \cdot \boldsymbol{\sigma}_{\mathrm{s}}-\rho_{\mathrm{s}} \phi \boldsymbol{g}-f_{\mathrm{f} \rightarrow \mathrm{s}}=0, \\
& \rho_{\mathrm{f}}(1-\phi) \frac{\mathrm{D}_{\boldsymbol{u}_{\mathrm{f}}} \boldsymbol{u}_{\mathrm{f}}}{\mathrm{D}_{\boldsymbol{u}_{\mathrm{f}}} t}-\nabla \cdot \boldsymbol{\sigma}_{\mathrm{f}}-\rho_{\mathrm{f}}(1-\phi) \boldsymbol{g}+f_{\mathrm{f} \rightarrow \mathrm{s}}=0, \\
& \frac{\partial \phi}{\partial t}+\nabla \cdot\left(\phi \boldsymbol{u}_{\mathrm{s}}\right)=0 \\
& \frac{\partial(1-\phi)}{\partial t}+\nabla \cdot\left[(1-\phi) \boldsymbol{u}_{\mathrm{f}}\right]=0
\end{aligned}
$$

Here the fields $\boldsymbol{u}$ (velocity), $\rho$ (density), and $\boldsymbol{\sigma}$ (Cauchy stress tensor) have values for both the porous medium and the liquid, indicated by their respective subscripts: "s" for the (solid) porous media and " $\mathrm{f}$ " for the fluid. The volume fraction of the solid in the porous material is given by $\phi$ (so $1-\phi$ gives the complementary non-solid fraction), and $\boldsymbol{g}$ represents any external forces, such as gravity. The operator $\frac{\mathrm{D}_{\boldsymbol{u}}}{\mathrm{D}_{\boldsymbol{u}} t}$ is the Eulerian material derivative under the flow velocity $\boldsymbol{u}$, defined as $\frac{\mathrm{D}_{u}}{\mathrm{D}_{\boldsymbol{u}} t}=\frac{\partial}{\partial t}+\boldsymbol{u} \cdot \nabla$. Lastly, $f_{\mathrm{f} \rightarrow \mathrm{s}}$ is the interaction force between the liquid and the solid porous medium. It is this force that we must derive to properly model wet cloth and yarn.

Equations (1a) and (1b) are the momentum equations of solid and fluid, respectively, while equations (1c) and (1d) are the corresponding laws of mass conservation (or continuity equations). We will elaborate below on the solid stress and interaction forces, including buoyancy and drag. But first, we must drop an assumption that we have made.

The continuity equations (1c) and (1d) assume that pores are fully filled with liquid, and thus the liquid volume in a unit material volume is given by $1-\phi$. How do we model a porous medium partially filled with liquid? One way to approach this is to (fully) saturate our porous medium with a fluid that represents both liquid and air components [Borja 2006].

Consider a fluid mixture of liquid and air. Since the air density is orders of magnitude smaller than the liquid density, we ignore the mass of the air. We assume that the fluid velocity field is shared by the liquid and air components moving in unison. We use the saturation variable $S_{\mathrm{r}}$ to indicate the volume fraction of liquid in the fluid (thus $1-S_{\mathrm{r}}$ indicates the volume fraction of air in the fluid). In such a mixture, the fluid density $\rho_{\mathrm{f}}$ (recall (1b)) becomes a fraction of the water density $\rho_{w}$ (i.e., $\rho_{\mathrm{f}}=S_{\mathrm{r}} \rho_{w}$ ). We can substitute this liquid-air fluid mixture, in place of only liquid, to obtain continuity equations that do not assume liquid saturation.

Unsaturated continuity equation. Consider a porous medium that is not necessarily (fully) saturated with liquid. Such a medium is (fully) saturated with our liquid-air fluid mixture. A unit volume of the porous fabric medium is the sum of three parts,

$$
\phi+(1-\phi) S_{\mathrm{r}}+(1-\phi)\left(1-S_{\mathrm{r}}\right)=1,
$$

where the three terms correspond to the volume fraction of solid, liquid, and air, respectively. The continuity equation (1d) of liquid can be modified to account for partial saturation using a slightly 


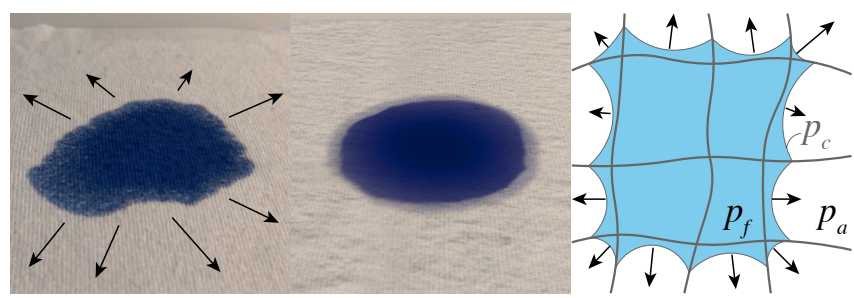

Fig. 3. Pore pressure example. Consider a piece of fabric laying on a table. The fabric is wet initially in a circular region. Near the boundary of the circle, the saturation $S_{\mathrm{r}}$ changes from zero to one, along the directions indicated by the arrows. On the left is a real photograph, and in the middle is our simulated result. $P_{\mathrm{c}}$ in this case is the pore pressure introduced by the water-air surface between the textile fibers (right). Because of the change of $S_{\mathrm{r}}$ along the radial directions, the gradient $\nabla\left(1-S_{\mathrm{r}}\right) P_{\mathrm{c}}$ in (8) generates interaction forces between the textile fibers and the water between. Macroscopically, these forces point along the directions of the arrows. Since the forces are mostly uniform in all directions, the fabric remains static, but the water spreads outwards.

different form,

$$
\frac{\partial(1-\phi) S_{\mathrm{r}}}{\partial t}+\nabla \cdot\left[(1-\phi) S_{\mathrm{r}} \boldsymbol{u}_{\mathrm{f}}\right]=0 .
$$

Lastly, subtracting (1d) from (1c) yields the incompressibility condition for the solid-fluid mixture,

$$
\nabla \cdot\left[\phi \boldsymbol{u}_{\mathrm{s}}+(1-\phi) \boldsymbol{u}_{\mathrm{f}}\right]=0 .
$$

In summary, equations (1a-1c) together with (3-4) form the mixture theory model for unsaturated porous media.

Solid stress. The effect of porosity on solid stresses is that, under the same deformation, the effective stress $\sigma_{\mathrm{s}}$ of a porous solid material is smaller than the corresponding stress $\sigma_{c}$ exhibited by a densely packed or non-porous material (i.e., with zero porosity). Given an applied deformation (or strain), $\sigma_{c}$ can be evaluated using a particular constitutive model, the choice of which depends on whether we are simulating wet cloth or yarn (see §4). The relationship between $\sigma_{c}$ and $\sigma_{\mathrm{s}}$ has been experimentally and numerically established by Makse et al. [2000], namely, $\sigma_{\mathrm{s}}=\phi^{\lambda} \sigma_{c}$, where the parameter $\lambda$ is material-dependent, usually taking values from $1 \sim 3$. In all our examples, we use the value $\lambda=2$.

Interaction forces. There are two relevant types of interaction forces between solid and liquid [Anderson and Jackson 1967]: the pressure gradient force $f_{\mathrm{f} \rightarrow \mathrm{s}}^{\mathrm{p}}$ and the drag force $f_{\mathrm{f} \rightarrow \mathrm{s}}^{\mathrm{d}}$. The total interaction force is

$$
f_{\mathrm{f} \rightarrow \mathrm{s}}=f_{\mathrm{f} \rightarrow \mathrm{s}}^{\mathrm{p}}+f_{\mathrm{f} \rightarrow \mathrm{s}}^{\mathrm{d}} .
$$

The pressure gradient acts when cloth and yarn are submerged (Figure 4). The drag force, on the other hand, is due to liquid-solid friction and wake turbulence. The next two subsections are dedicated to our derivation of the specific forms of these forces for wet cloth and yarn.

\subsection{Pressure Gradient}

In a saturated solid-liquid mixture, the pressure gradient is

$$
f_{\mathrm{f} \rightarrow \mathrm{s}}^{\mathrm{p}}=-\phi \nabla p \text {. }
$$

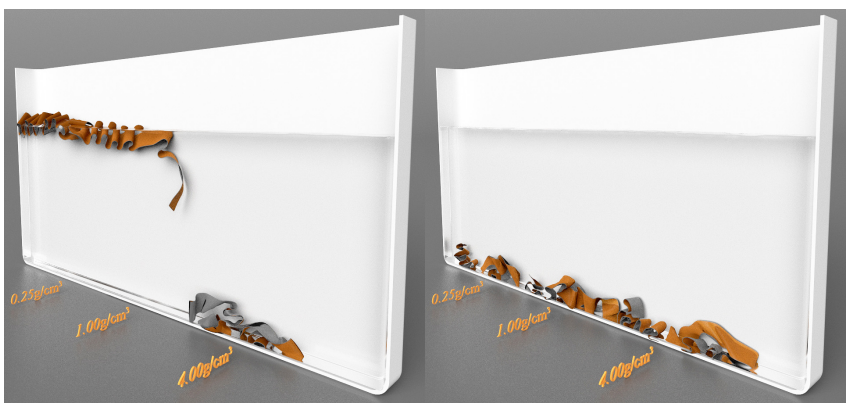

Fig. 4. Comparison with and without the liquid pressure gradient applied to solid. A simulation with the liquid pressure gradient applied to cloth yields correct buoyancy (left), where cloth lighter than water floats and cloth heavier than water sinks. Without the pressure gradient, all the cloth erroneously sinks (right).

Here we neglect the liquid stress induced by the porous solid, a standard assumption for open pores [Pitman and Le 2005]. The liquid pressure $p$ is smoothly varying except for a jump at the liquid free surface induced by surface tension.

An unsaturated porous medium has tiny air pockets, for which the surface tension force exactly balances the liquid-air pressure jump. The myriad air pockets make for a markedly more complex liquid surface, since air and liquid are present "everywhere;" the jumps due to surface tension are densely distributed and more appropriately captured in a homogenized force balance, $p_{\mathrm{f}}-p_{\mathrm{a}}=p_{\mathrm{c}}$, referring to the liquid, air, and pore pressure, respectively [Borja 2006].

From mixture theory, the effective pressure $p$ of an unsaturated porous medium is given by weighting the component pressures by the saturation $S_{\mathrm{r}}$ [Borja 2006],

$$
p=S_{\mathrm{r}} p_{\mathrm{f}}+\left(1-S_{\mathrm{r}}\right) p_{\mathrm{a}} .
$$

Substituting the force balance $p_{\mathrm{f}}-p_{\mathrm{c}}=p_{\mathrm{a}}$ into (7), then into (6),

$$
f_{\mathrm{f} \rightarrow \mathrm{s}}^{\mathrm{p}}=-\phi \nabla p=\underbrace{-\phi \nabla p_{\mathrm{f}}}_{\text {buoyancy }}+\underbrace{\phi \nabla\left(\left(1-S_{\mathrm{r}}\right) p_{\mathrm{c}}\right)}_{\text {pore pressure }} .
$$

The first term of the pressure gradient governs buoyancy, the force that pushes lighter objects up toward the fluid surface. As we can see, the second term is present only for an unsaturated porous medium $\left(S_{\mathrm{r}}<1\right)$. We now explore this pore pressure term.

Suction tensor. It has been confirmed by experimental studies [Scholtès et al. 2009] that pore pressure depends on the porous solid microstructure. Here, we develop a pore pressure model suited to our application.

The void space between textile fibers, which is oriented along individual yarns, yields an anisotropic microstructure. Consequently, our pore pressure is also anisotropic, and must therefore be described by a second-order tensor rather than a scalar. This tensor is called the suction tensor in the mechanics literature.

Drawing on the literature on porous flow through fibers, we propose a model for the suction tensor specialized to the case of cloth, yarn, and combinations of the two. Consider a pack of fibers along a yarn segment (Figure 5-a). When mixed with water the void spaces between individual fibers effectively form capillary tubes that act to transport water. The pore (or suction) pressures along 


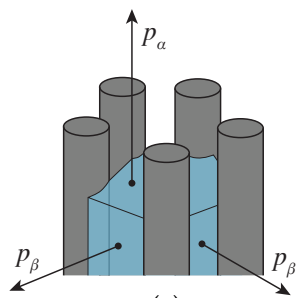

(a)

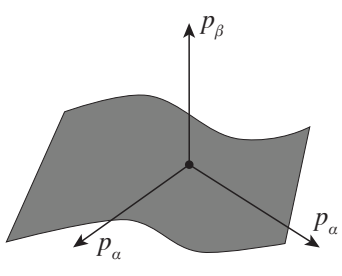

(b)

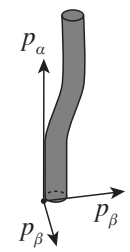

(c)

Fig. 5. Fiber pack, cloth and yarn orientation. In our derivation of the pressure gradient and drag forces, we use a canonical frame of reference to orient the fiber pack, cloth, and yarn. Cloth and yarn in arbitrary orientations are first rotated into this frame of reference to compute the force tensors, and then rotated back to their original frame.

the fiber direction and the perpendicular direction are, respectively,

$$
p_{\alpha}=\frac{2 \phi \gamma \cos \theta}{(1-\phi) r_{b}} \text { and } p_{\beta}=\frac{p_{\alpha}}{2},
$$

as Masoodi and Pillai [2012a] introduced and experimentally verified. Here $\phi$ is again the volume fraction of the capillary tubes (in our case the textile fibers), $\gamma$ is the surface tension coefficient of liquid (i.e., $\gamma=72.0 \mathrm{dyn} / \mathrm{cm}$ for water), $\theta$ is the equilibrium contact angle between liquid and the fibers, and $r_{b}$ is the radius of the capillary tubes.

Adapting this concept to our setting, we note that if a yarn segment is aligned along the $Z$-direction, we can write its suction tensor as a diagonal matrix whose diagonal elements are $\left[p_{\beta} p_{\beta} p_{\alpha}\right]$. Similarly, in a small piece of cloth with its normal aligned along the $Z$-direction (Figure 5-b), the textile fibers are instead oriented along the $X$ - and $Y$-directions. Then, the suction tensor is another diagonal matrix with diagonal elements $\left[p_{\alpha} p_{\alpha} p_{\beta}\right]$. When individual yarn strands extend perpendicularly from the cloth surface (Figure 5-c) - for example, when simulating a fuzzy towel - we express the suction tensor as a weighed combination of both diagonal matrices,

$$
\hat{\mathbf{P}}_{c}=s_{\mathrm{f}}\left[\begin{array}{ccc}
p_{\alpha} & 0 & 0 \\
0 & p_{\alpha} & 0 \\
0 & 0 & p_{\beta}
\end{array}\right]+\left(1-s_{\mathrm{f}}\right)\left[\begin{array}{ccc}
p_{\beta} & 0 & 0 \\
0 & p_{\beta} & 0 \\
0 & 0 & p_{\alpha}
\end{array}\right] \text {, }
$$

where we call $s_{\mathrm{f}}$ the "shape fraction": when we consider the suction tensor in an infinitesimal region of cloth or yarn, $s_{\mathrm{f}}=0$ if this region is occupied entirely by a yarn strand, $s_{\mathrm{f}}=1$ if it is entirely occupied by cloth, and $s_{\mathrm{f}}$ lies between 0 and 1 if the region is near the root of a yarn strand extending from a piece of cloth (Figure 5-c).

With the suction tensor $\hat{\mathbf{P}}_{\mathrm{c}}$ defined for cloth and yarn in the canonical orientation above, the suction tensor $\mathbf{P}_{c}$ in an arbitrary orientation will be a rotated version of $\hat{\mathbf{P}}_{\mathrm{c}}$, namely

$$
\mathbf{P}_{\mathrm{c}}=\mathbf{R}^{T} \hat{\mathbf{P}}_{\mathrm{c}} \mathbf{R} \text {. }
$$

In cloth, $\mathbf{R}$ is the rotation matrix that rotates the cloth normal to the $\mathrm{Z}$-direction, and in yarn, $\mathrm{R}$ rotates the yarn tangent to the $\mathrm{Z}$ direction. (In the mixtures we described above, these directions are mutually aligned.)

Finally, having developed our new application-specific definition of the suction tensor, the pressure gradient force in (8) can be rewritten as

$$
f_{\mathrm{f} \rightarrow \mathrm{s}}^{\mathrm{p}}=-\phi \nabla p_{f}+\phi \nabla \cdot\left(\left(1-S_{\mathrm{r}}\right) \mathbf{P}_{\mathrm{c}}\right)
$$

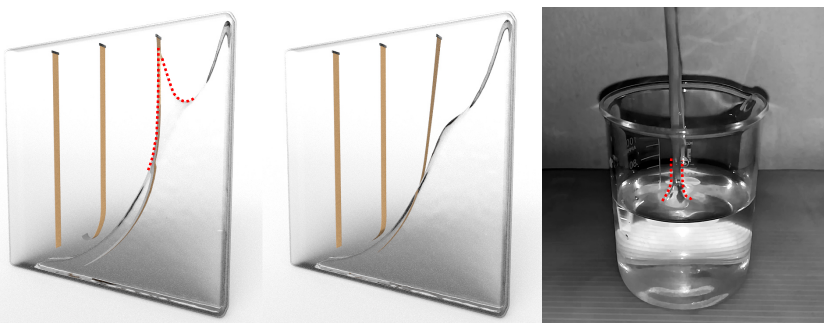

Fig. 6. Comparison between nonlinear and linear drag models. Nonlinear drag (left) exhibits a sharp "kink" (red dashed curve) around the liquid-solid interface due to fast-moving cloth having pulled water with it. Since linear drag is not suitable for high Reynolds number flows, this effect is not seen for linear drag (middle). This effect can, however, be readily observed in physical experiments (right, with red dashed curve).

where the divergence of our anisotropic suction (stress) tensor has taken the place of the gradient of the scalar pore pressure.

Remark. The total stress, an oft-used quantity when modeling porous materials such as sand and soil [Song and Borja 2014], is the sum of the solid stress $\sigma_{\mathrm{s}}$ and fluid stress $\sigma_{\mathrm{f}}$. The equation above effectively states that $\sigma_{\mathrm{f}}=-p_{\mathrm{f}} \mathbf{I}_{3}+\left(1-S_{\mathrm{r}}\right) \mathbf{P}_{\mathrm{c}}$, where $\mathbf{I}_{3}$ is a $3 \times 3$ identity. In $\S 3.1$, we saw that $\sigma_{\mathrm{s}}=\phi^{\lambda} \sigma_{c}$. Our total stress is therefore $\phi^{\lambda} \boldsymbol{\sigma}_{\mathrm{c}}-p_{\mathrm{f}} \mathbf{I}_{3}+\left(1-S_{\mathrm{r}}\right) \mathbf{P}_{\mathrm{c}}$. For saturated and densely packed porous material $\left(\lambda \rightarrow 0\right.$ and $\left.S_{\mathrm{r}} \rightarrow 1\right)$, our definition of total stress becomes $\sigma_{\mathrm{c}}-p_{\mathrm{f}} \mathbf{I}_{3}$, which is precisely consistent with the classic Terzaghi's total stress [Terzaghi 1943].

\subsection{Drag Force}

Drag. Liquid flow through a porous solid is resisted by a drag force proportional to the relative velocity, $f_{\mathrm{f} \rightarrow \mathrm{s}}^{\mathrm{d}}=\tilde{\mathrm{C}}\left(\boldsymbol{u}_{\mathrm{f}}-\boldsymbol{u}_{\mathrm{s}}\right)$, where $\tilde{\mathrm{C}}$ is a diagonal matrix of drag coefficients, $\hat{C}_{i}$.

For a pack of fibers oriented along the $\mathrm{Z}$ axis (Figure 5), the drag coefficients along the transversal $(1 \leq i \leq 2)$ and lateral $(i=3)$ directions can be expressed in terms of anisotropic permeability.

Permeability is a measure of the ability of a porous material to allow liquids to pass through it [Bear 2013]. The permeabilities of flows lateral and transverse to a pack of fibers are [Stylianopoulos et al. 2008]

$$
\begin{aligned}
& k_{\alpha}=\frac{-\ln \phi-1.476+2 \phi-0.5 \phi^{2}}{16 \phi} d^{2} \text { and } \\
& k_{\beta}=\frac{-\ln \phi-1.476+2 \phi-1.774 \phi^{2}+4.078 \phi^{3}}{32 \phi} d^{2},
\end{aligned}
$$

respectively, in terms of the volume fraction $\phi$ and fiber diameter $d$.

Drag coefficient. Yazdchi and Luding [2012] relate the drag coefficient to the permeability of a fibrous material. The drag coefficient $\hat{C}_{i}$ is normalized by the liquid viscosity $\mu$ and fiber diameter $d$ to define a dimensionless drag, or modified friction factor, $f_{i}=-d^{2} \hat{C}_{i} / \mu$. Similarly, the permeability is normalized as $K_{i}=k_{i} d^{2}$. The dimensionless drag and permability are related via

$$
-f_{i}=\frac{1}{K_{i}}+\chi_{i} \operatorname{Re}_{i}^{c}
$$


where the exponent $c=1.6$ is a constant; $\operatorname{Re}_{i}=\rho_{\mathrm{f}}\left(\boldsymbol{u}_{\mathrm{f}, i}-\boldsymbol{u}_{\mathrm{s}, i}\right) d / \mu$ is the Reynolds number, where the subscript $i$ of $\boldsymbol{u}_{\mathrm{f}}$ and $\boldsymbol{u}_{\mathrm{s}}$ indicates the $X$-, $Y$-, or $Z$-component of the velocity.

The coefficient $\chi_{i}$ weights the nonlinear second term relative to the linear first term. Many models exist for computing $\chi_{i}$, and we choose to use the classic Ergun equation [Ergun 1952], as validated by Yazdchi and Luding [2012]. Replacing the various quantities in (14), we obtain our final formula for the drag coefficient,

$$
\hat{C}_{i}=\frac{\mu}{k_{i}}+\frac{1.75}{\sqrt{150}} \frac{\rho_{\mathrm{f}}^{c} d^{c-1} \mu^{1-c}}{(1-\phi)^{\frac{3}{2}} \sqrt{k_{i}}}\left\|\boldsymbol{u}_{\mathrm{f}}-\boldsymbol{u}_{\mathrm{s}}\right\|_{2}^{c}
$$

These coefficients allow us to compute the drag force when a pack of fibers are oriented along the Z-direction. Given cloth or yarn with an arbitrary orientation, we construct a rotated drag tensor $\mathrm{C}$ in a similar manner to the suction tensor in (11): namely, $\mathbf{C}=\mathbf{R}^{T} \hat{\mathbf{C}} \mathbf{R}$, where $\hat{\mathrm{C}}$ is a diagonal matrix. For cloth, $\hat{\mathrm{C}}$ has the diagonal elements $\left[\hat{C}_{\beta} \hat{C}_{\beta} \hat{C}_{\alpha}\right.$ ], while in yarn $\hat{\mathbf{C}}$ has the diagonal elements $\left[\hat{C}_{\alpha} \hat{C}_{\alpha} \hat{C}_{\beta}\right]$. Here $\hat{C}_{\alpha}$ and $\hat{C}_{\beta}$ are determined by substituting $k_{\alpha}$ and $k_{\beta}$ of (13) into the $k_{i}$ of (15), respectively. In general, $\hat{\mathrm{C}}$ is a combination of the two cases, defined in the same way as in (10). $\mathbf{R}$ is a rotational matrix that aligns the cloth's normal direction or the yarn's tangential direction with the Z-direction, as in (11).

Finally, the drag force is computed as

$$
f_{\mathrm{f} \rightarrow \mathrm{s}}^{\mathrm{d}}=\mathrm{C}\left(\boldsymbol{u}_{\mathrm{f}}-\boldsymbol{u}_{\mathrm{s}}\right)=\mathbf{R}^{T} \hat{\mathrm{C}} \mathrm{R}\left(\boldsymbol{u}_{\mathrm{f}}-\boldsymbol{u}_{\mathrm{s}}\right) .
$$

Remark I. Dimensional analysis provides a useful sanity check on our derivation. The value $\hat{C}_{i}$ in (15) has units of $\mathrm{g} \cdot \mathrm{cm}^{-3} \cdot \mathrm{s}^{-1}$ for any positive $c$ value. Therefore, $f_{\mathrm{f} \rightarrow \mathrm{s}}^{\mathrm{d}} / \rho_{\mathrm{s}}$ always has units of $\mathrm{cm} \cdot \mathrm{s}^{-2}$, which are precisely the units of acceleration.

Remark II. Drag force models have been used in many computer graphics simulations, yet almost all such models have been linear with respect to the relative velocity. For example, recent work on simulating sand and water mixtures [Tampubolon et al. 2017] adopts a linear model. Meanwhile, studies in porous mechanics have shown that the drag force is nonlinear, especially when the Reynolds number is not small [Masoodi and Pillai 2012a]. In Figure 6 and our supplemental video, we compare our nonlinear drag model in (15) with the linear drag model that ignores the second term of (15), to demonstrate their very distinct visual difference.

\subsection{Dynamic and Quasi-Static Model}

Dynamic model. Putting together all the forces derived above, our mixture model for cloth and yarn is comprised of five equations: namely, the momentum equations (1a) and (1b); the continuity equation (1c) for solid material; the continuity equation (3) for the liquid, which also advects the porous saturation $S_{r}$; and the incompressibility condition (4). To complete (1a) and (1b), the interaction force $f_{\mathrm{f} \rightarrow \mathrm{s}}$ is the sum of the pressure gradient force (12) and the drag force (16). We refer to these equations as the dynamic equations of wet cloth and yarn. In the next section, we will numerically solve them by discretizing the entire domain of the liquid, wet cloth, and wet yarn using Eulerian grids.
Quasi-static model. To capture liquid diffusing and convecting within the thin volume of cloth and yarn, directly discretizing the dynamic equations in 3D necessitates the use of very fine grids, resulting in prohibitive simulation costs. We therefore treat this case specially. We observe that water travels along the cloth and yarn volume slowly even while the cloth and yarn might undergo large deformation. This suggests that we can model the liquid motion in the frame of reference attached to the cloth or yarn. In this frame of reference, the Reynolds number is relatively low, so we choose to model the liquid motion quasi-statically as diffusion on the codimensional objects (i.e., 2D surface for cloth and 1D curve for yarn). In particular, we ignore the inertia term in (1b). We further note that the cloth and yarn material is isotropic in the codimensional space, and hence so is the pressure tensor. Then, Equation (1b) after substituting (12) and (16) can be simplified into

$$
\frac{1}{\rho_{\mathrm{f}}} \nabla\left[\left(1-S_{\mathrm{r}}\right) p_{\alpha}-p_{\mathrm{f}}\right]-\frac{\hat{C}_{\alpha}}{\rho_{\mathrm{f}}(1-\phi)}\left(\boldsymbol{u}_{\mathrm{f}}-\boldsymbol{u}_{\mathrm{s}}\right)+\tilde{\boldsymbol{g}}=0 .
$$

Because the frame of reference is non-inertial, the force $\tilde{\boldsymbol{g}}$ must now include not only the external force $\boldsymbol{g}$ but also additional fictitious forces, such as the centrifugal and Coriolis forces (to be discussed further in $\S 4)$.

Equation (17) allows us to express $\boldsymbol{u}_{\mathrm{f}}$ with respect to $\boldsymbol{u}_{\mathrm{s}}, p_{\mathrm{f}}$, and $p_{\alpha}$, by isolating $\boldsymbol{u}_{\mathrm{f}}$ on one side of the equation. Substituting it in (3) yields the equation to be solved in the codimensional space:

$$
\frac{\partial \epsilon_{L}}{\partial t}+\nabla \cdot\left[\frac{(1-\phi) \epsilon_{L}\left(\nabla\left(\tilde{p}_{\alpha}-p_{\mathrm{f}}\right)+\rho_{\mathrm{f}} \tilde{\boldsymbol{g}}\right)}{\hat{C}_{\alpha}}+\epsilon_{L} \boldsymbol{u}_{\mathrm{s}}\right]=0 .
$$

where $\tilde{p}_{\alpha}=\left(1-S_{\mathrm{r}}\right) p_{\alpha}$, and we define $\epsilon_{L}=(1-\phi) S_{\mathrm{r}}$ as the volume fraction of liquid in the unsaturated mixture. This is a convectiondiffusion equation describing how $\epsilon_{L}$ is transported quasi-statically along cloth surfaces and yarn strands.

Remark. If we ignore external forces and the pressure from the bulk fluid, and assume the porous solid is static, then $p_{\mathrm{f}}, \tilde{\boldsymbol{g}}$, and $\boldsymbol{u}_{\mathrm{s}}$ in (18) all vanish, while $\phi$ remains constant. Then this equation reduces to the famous Richards equation in soil mechanics [Richards 1931], that describes the movement of water in unsaturated soils:

$$
\frac{\partial S_{\mathrm{r}}}{\partial t}=\nabla \cdot\left[\tilde{D}\left(S_{\mathrm{r}}\right) \nabla S_{\mathrm{r}}\right],
$$

where $\tilde{D}\left(S_{\mathrm{r}}\right)$ is called the diffusivity, and is usually some function of $S_{\mathrm{r}}$ and the permeability. In our model, $\tilde{D}\left(S_{\mathrm{r}}\right)=\frac{(1-\phi) p_{\alpha} S_{\mathrm{r}}}{\hat{C}_{\alpha}}$, which has a linear dependence on $S_{\mathrm{r}}$ and corresponds to a linear water retention curve. Other popular models, such as Brooks-Corey [1964], van Genutchen [1980], or models from experimental data fitting [Landeryou et al. 2005], usually assume an infinite suction pressure when $S_{\mathrm{r}}$ approaches zero. We adopt a linear model since it is effective and numerically stable, and can be derived from a standard modification of mixture theory for unsaturated porous media.

\section{NUMERICAL SIMULATION}

Having laid down the governing equations, we turn our attention to the numerics. 


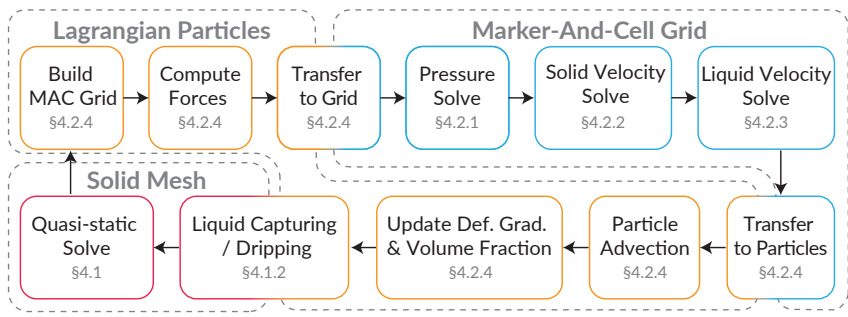

Fig. 7. Overview of our numerical method.

Method overview. We discretize the quasi-static equation (18) over Lagrangian fabric "solid" meshes, and the remaining dynamic equations over a "background" Eulerian marker-and-cell (MAC) grid augmented with Lagrangian particles for advection.

Bulk liquid is simulated using the affine particle-in-cell (APIC) method [Jiang et al. 2015]. Each Lagrangian liquid particle carries a scalar volume and two set of velocities: the liquid velocity $\boldsymbol{u}_{\mathrm{f}}$ and the solid porous material's velocity $\boldsymbol{u}_{\mathrm{s}}$.

The porous fabric solid is simulated using a Lagrangian mesh, with each vertex carrying the solid velocity $\boldsymbol{u}_{\mathrm{s}}$, a porous volume fraction $\phi$, and a liquid saturation fraction $S_{\mathrm{r}}$. We use the APIC method to distribute data from Lagrangian points (liquid particles and solid mesh vertices) to the Eulerian grid faces, and vice versa.

The elastic forces of the fabric are computed using discrete shells [Grinspun et al. 2003] for woven cloth, and discrete elastic rods [Bergou et al. 2010; Kaldor et al. 2008] for knitted garments. These elastic forces are coupled with the background grid using the method of Jiang et al. [2017] to resolve collision and frictional forces.

At each timestep, our method performs the following steps (see Figure 7 for a visual overview of our algorithm):

(1) Build the MAC grid (§4.2.4)

(2) Compute solid internal forces and apply the flow-rule for solid plasticity (§4.2.4),

(3) Map liquid and solid particles onto the Eulerian grid (§4.2.4),

(4) Solve the pressure projection ( $\$ 4.2 .1)$,

(5) Solve the solid velocity $(\S 4.2 .2)$,

(6) Update the liquid velocity (§4.2.3),

(7) Map the liquid and solid velocity back to particles, update solid deformation gradient, advect particles (§4.2.4),

(8) Handle liquid capture and dripping for cloth and yarn (§4.1.2),

(9) Solve the quasi-static equation on solid meshes (§4.1).

\subsection{Codimensional Quasi-Static Simulation}

Because fabric strand features are $\sim 4-8 \times$ smaller than a grid cell, we solve (18) on the Lagrangian meshes directly without relying on the MAC grid. We must consider three types of mesh configurations: (woven) cloth triangles, (individual or knitted) yarn segments, and the junctions between them (see Figure 8). Junctions are useful not only for modeling cloth-knit assemblies, but also other non-manifold structures, such as fuzzy fabric (see Figure 13).

Notation. The subscript $\mathbf{E}$ indicates that a field is discretized over mesh elements, triangle faces and yarn segments, for example, $\boldsymbol{u}_{\mathrm{s}, \mathrm{E}}$ represents solid velocity defined on elements. By contrast, the subscript $\mathbf{V}$ indicates that a field is discretized over vertices. Individual

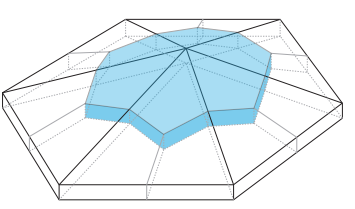

(a)

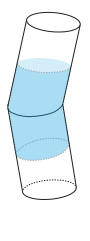

(b)

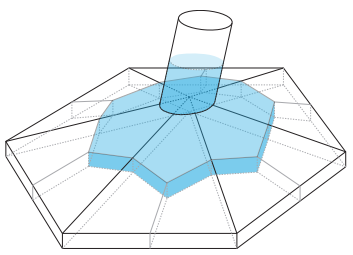

(c)
Fig. 8. Codimensional objects. (a) Cloth modeled as a triangle mesh. (b) Yarn modeled as a sequence of cylinders. (c) A cloth-yarn joint. The volume of the shaded region is used to compute vertex weights. Each triangle is uniformly divided according to its barycenter and edge bisectors.

timesteps are indicated by a superscript $n$ (e.g., $p_{\mathrm{V}}^{n}$ for pressure stored on vertices at timestep $n$ ), and $h$ always denotes the timestep size.

4.1.1 Codimensional solve. To solve (18) on irregular meshes, we first define the necessary mesh-based discrete differential operators [Botsch et al. 2010]. We will use uppercase sans serif letters (e.g., G) to denote discrete operators.

Each mesh element (cloth triangle or rod segment) is associated with a time-invariant finite volume $V_{\mathrm{E}}$ in physical space. For a cloth triangle, this is computed from its undeformed area and fabric thickness. For a rod segment, this is computed from its undeformed length and yarn thickness.

Each vertex is also associated with a time-invariant finite volume $V_{\mathrm{V}}$ (Figure 8), computed in a typical barycentric style: incident cloth triangles contribute a third of their volume to each vertex, and incident rod segments contribute half of their volume to each vertex.

The liquid volume discretized on vertices is given by the vector $V_{\mathrm{f}, \mathrm{V}}=\left([1-\phi]_{\mathrm{V}}\right) S_{r, \mathrm{~V}} V_{\mathrm{V}}$, where $[1-\phi]_{\mathrm{V}}$ and $S_{r, \mathrm{~V}}$ are the liquid volume fraction and liquid saturation per vertex (recall §3.1). Similarly, $V_{\mathrm{f}, \mathrm{E}}$ is a vector describing the liquid volume per element. With these expressions, we can discretize the convection-diffusion equation (18) for the liquid fraction using implicit integration as

$$
\begin{aligned}
& V_{\mathrm{f}, \mathrm{V}}^{n+1}=V_{\mathrm{f}, \mathrm{V}}^{n}-h G^{T} {\left[\hat { C } _ { \alpha , \mathrm { E } } ^ { - 1 } \left([1-\phi]_{\mathrm{E}}[V]_{\mathrm{f}, \mathrm{E}}^{n+1}\right.\right.} \\
&\left.\left.\left(\mathrm{G}\left[\tilde{p}_{\alpha}-p_{\mathrm{f}}\right]_{\mathrm{V}}^{n+1}+\rho_{\mathrm{f}} \mathbf{g}_{\mathrm{E}}\right)\right)+[V]_{\mathrm{f}, \mathrm{E}}^{n+1} \boldsymbol{u}_{\mathrm{s}, \mathrm{E}}^{n+1}\right],
\end{aligned}
$$

where the notation $[\cdot]$ denotes the operator that converts a vector into a diagonal matrix.

On triangle meshes, we use the standard gradient and divergence operators described in detail by Botsch et al. [2010]. The gradient operator $G \in \mathbb{R}^{3|\mathcal{E}| \times|\mathcal{V}|}$ maps the vector form of a quantity defined on vertices to its gradient on elements, and its adjoint, the divergence operator $\mathrm{G}^{T} \in \mathbb{R}^{|\mathcal{V}| \times 3|\mathcal{E}|}$, maps a vector quantity on elements to its divergence on vertices. $|\mathcal{V}|$ and $|\mathcal{E}|$ indicate the total number of vertices and elements, respectively. Construction of $G$ relies on the same weight contributions used to compute $V_{\mathrm{V}}$.

This is a system of equations with respect to $V_{\mathrm{f}, \mathrm{V}}^{n+1}$ that is nonlinear, and, in the general case, very difficult to solve [Paniconi et al. 1991]. In our case, the Reynolds number of liquids flowing through cloth and yarn is low, so $V_{\mathrm{f}, \mathrm{V}}^{n+1}$ remains fairly close to $V_{\mathrm{f}, \mathrm{V}}^{n}$ over a timestep. Thus, we solve (20) using fixed-point iterations [Burden and Faires 1985]: in each iteration, we update $V_{\mathrm{f}, \mathrm{E}}^{n+1}$ by interpolating 


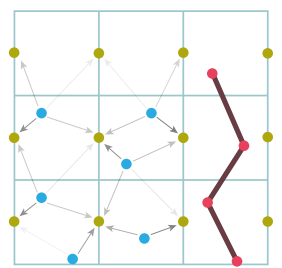

(a)

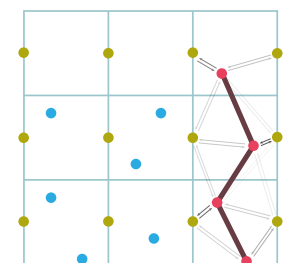

(b)

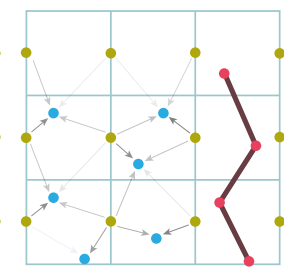

(c)
Fig. 9. Liquid capturing. • liquid particles; • solid vertices; • grid faces. Opacity indicates kernel weight. For legibility, only the faces in the $x$-axis are shown; similar operations are done for the $y$ - and $z$-axis. (a) Liquid volume from particles is distributed onto grid faces. (b) Solid vertices absorb volume from grid faces whose volume is reduced correspondingly. (c) The retained volume is stored back onto particles.

$V_{\mathrm{f}, \mathrm{V}}^{n+1}$ from the previous iteration, and then update $V_{\mathrm{f}, \mathrm{V}}^{n+1}$ using (20). In practice, this method converged within four iterations for the scenes we tested.

4.1.2 Liquid capturing and dripping. When cloth and yarn come in contact with water they begin to absorb it, and become wet. On the other hand, if cloth or yarn becomes locally oversaturated, water starts to drip off. Therefore, our codimensional simulation must also exchange liquid with the background fluid grid.

Notation. We use a subscript $p$ to indicate quantities stored at liquid particles, and subscript $i$ to indicate quantities on the grid (i.e., grid faces). For example, $V_{f, p}^{n}$ is the volume of a liquid particle $p$ at timestep $n$.

Water absorption is performed with the following steps (see figure 9):

(1) For each grid face, we update its liquid volume by summing contributions from liquid particles in the grid, $V_{\mathrm{f}, i}^{n}=$ $\sum_{p} V_{\mathrm{f}, p}^{n} w_{p, i}$, where $w_{p, i}$ is the kernel function between the particle $p$ and grid face $i$, as defined by Jiang et al. [2015].

(2) Each solid vertex (cloth or yarn) captures liquid by taking liquid volume contributions from the background grid: $V_{\mathrm{f}, \mathrm{V}}^{n}=$ $\sum_{i} V_{\mathrm{f}, i}^{n} w_{i, \mathrm{~V}}$, where $w_{i, \mathrm{~V}}$ is the kernel function between grid face $i$ and solid vertex $\mathbf{V}$.

(3) For each grid face, we compute the amount of liquid removed by the cloth or yarn: $V_{\mathrm{f}, i}^{-}=\sum_{\mathrm{V}} V_{\mathrm{f}, i}^{n} w_{i, \mathrm{~V}}$, which totals the face's liquid contribution to all solid vertices.

(4) Lastly, we update each liquid particle's volume by computing the weighted sum of the updated liquid volume on grid faces: $V_{\mathrm{f}, p}^{n+1}=\left(\sum_{i}\left(V_{\mathrm{f}, i}^{n}-V_{\mathrm{f}, i}^{-}\right) w_{p, i}\right) /\left(\sum_{i} \sum_{\mathrm{V}} w_{p, i} w_{i, \mathrm{~V}}\right)$.

The updated volume of liquid particles will be used in the next timestep of the grid simulation (see §4.2).

Liquid drips off of cloth or yarn when a solid vertex is oversaturated. This is indicated by the condition $V_{\mathrm{f}, \mathrm{V}}>V_{\mathrm{V}}\left(1-\phi_{\mathrm{V}}\right)$. If it is satisfied, we inject liquid particles back into the grid. Each generated liquid particle has a fixed volume $V_{r}{ }^{1}$. The number of liquid particles

${ }^{1}$ We follow a standard rule of thumb to set $V_{r}$. As discussed by Um et al. [2017], a common practice is to have eight particles in a grid cell, each having sufficient volume to cover half of the cell size in each dimension. This means that $V_{r}=\frac{\pi \sqrt{3} \delta x^{3}}{16}$ where $\delta x$ is the size of a grid cell. that an oversaturated vertex can generate is $N_{d}=\left\lfloor\frac{V_{\mathrm{f}, \mathrm{V}}-V_{\mathrm{V}}\left(1-\phi_{p}\right)}{V_{r}}\right\rfloor$. We then uniformly sample $N_{d}$ positions on the elements (triangles and yarn segments) incident to the vertex, placing a liquid particle at each. Since liquid flow on the cloth and yarn is assumed to be quasi-static, the velocity of a new particle is set to the solid velocity at its position. Afterward, the liquid volume at the oversaturated solid vertex is updated to $V_{\mathrm{f}, \mathrm{V}}-N_{d} V_{r}$.

\subsection{Grid Simulation}

We solve the dynamic equations of wet cloth and yarn on the MAC grid. First, we discretize the incompressibility condition (4) and obtain

$$
h \mathrm{G}^{T}[\phi] \boldsymbol{u}_{\mathrm{s}}^{n+1}+h \mathrm{G}^{T}[1-\phi] \boldsymbol{u}_{\mathrm{f}}^{n+1}=0 .
$$

Here we reuse $\mathrm{G}$ and $\mathrm{G}^{T}$ to denote the (finite-volume) gradient and divergence operators, analogous to those in (20), but on the grid.

When discretizing the momentum equations (1a) and (1b), we ignore the advection terms (i.e., the $\boldsymbol{u} \cdot \nabla$ term in the material derivative), because we advect the liquid and solid materials in a separate substep via background particles with the APIC method (to be discussed in §4.2.4). Moreover, since cloth and yarn can often be highly stiff, they demand an implicit discretization of (1a). Otherwise, very small timestep sizes are needed, which would dramatically slow down the simulation. Thus, discretizing the momentum equations (1a) and (1b) yields

$$
\begin{aligned}
& {\left[\left(\mathbf{M}_{\mathrm{s}}+h[\mathbf{C}] \mathbf{V}_{\mathrm{c}}\right)+h^{2} \mathbf{H}\right] \boldsymbol{u}_{\mathrm{s}}^{n+1}-h[\mathbf{C}] \mathbf{V}_{\mathrm{c}} \boldsymbol{u}_{\mathrm{f}}^{n+1}+h \mathbf{V}_{\mathrm{s}} \mathbf{G} \boldsymbol{p}^{n+1}} \\
& =h f_{\mathrm{s}}+\mathbf{M}_{\mathrm{s}} \boldsymbol{u}_{\mathrm{s}}^{n} \text {, } \\
& \left(\mathbf{M}_{\mathrm{f}}+h[\mathrm{C}] \mathbf{V}_{\mathrm{c}}\right) \boldsymbol{u}_{\mathrm{f}}^{n+1}-h[\mathrm{C}] \mathbf{V}_{\mathrm{c}} \boldsymbol{u}_{\mathrm{s}}^{n+1}+h \mathbf{V}_{\mathrm{f}} \mathrm{G} p^{n+1}=h f_{\mathrm{f}}+\mathbf{M}_{\mathrm{f}} \boldsymbol{u}_{\mathrm{f}}^{n}
\end{aligned}
$$

where $\mathbf{M}_{\mathrm{s}}, \mathbf{M}_{\mathrm{f}}, \mathbf{V}_{\mathrm{c}}, \mathbf{V}_{\mathrm{s}}$, and $\mathbf{V}_{\mathrm{f}}$ are all diagonal matrices. We obtain $\mathbf{M}_{\mathrm{s}}$ by distributing the mass of cloth and yarn vertices to the face centers of grid cells (see §4.2.4). We obtain $\mathbf{V}_{\mathrm{c}}$ similarly by distributing vertex volumes $V_{\mathrm{V}}$ to the face centers of grid cells. Since a vertex volume is occupied by solid, liquid, and air, $\mathbf{V}_{\mathrm{s}}=[\phi] \mathbf{V}_{\mathrm{c}}$ is the solid portion of $\mathbf{V}_{\mathrm{c}}$, while $\mathbf{V}_{\mathrm{f}}=[1-\phi]\left[S_{\mathrm{r}}\right] \mathbf{V}_{\mathrm{c}}$ is the liquid portion of $\mathbf{V}_{\mathrm{c}}$. $\mathbf{M}_{\mathrm{f}}$ is the mass matrix of liquid. [C] is a tridiagonal matrix whose $3 \times 3$ diagonal subblocks are the drag tensors (as defined in (16)) evaluated at grid face centers. Lastly, $f_{\mathrm{s}}$ includes forces on solid vertices and are distributed to the grid's face centers, $f_{\mathrm{f}}$ are forces applied on the liquid, and $\mathbf{H}$ is the Jacobian matrix of solid force $f_{\mathrm{s}}$ with respect to the solid vertex positions. Their specific forms will be given in $\$ 4.2 .4$.

Assembling the discrete equations (21-23), we obtain a system of linear equations with respect to the unknowns $\boldsymbol{u}_{\mathrm{s}}^{n+1}, \boldsymbol{u}_{\mathrm{f}}^{n+1}$, and $p^{n+1}$. However, solving this linear system is rather challenging since it is large and unsymmetric. It couples $\boldsymbol{u}_{\mathrm{s}}^{n+1}, \boldsymbol{u}_{\mathrm{f}}^{n+1}$, and $\boldsymbol{p}^{n+1}$ together, and its size is about seven times the number of grid faces, which makes direct solvers impractical. To make matters worse, the linear system can be stiff due to the large stretching stiffness of cloth and yarn or large pressure gradient applied, thus requiring many iterations for iterative solvers to converge to the solution. We initially attempted to use BiCGSTAB, but it successfully converged only under impractically small time step sizes (Courant number less 
than $10^{-5}$ ). We therefore propose an efficient alternative solution strategy.

Solver overview. We begin by summarizing the three main steps of our solver. First, by discretizing (1a) explicitly, we reduce the linear system to a smaller one involving $\boldsymbol{p}^{n+1}$ alone. After obtaining $\boldsymbol{p}^{n+1}$, we return to an implicit discretization of (1a), and solve another system of linear equations with respect to $\boldsymbol{u}_{\mathrm{s}}^{n+1}$ alone. Lastly, we construct a linear system to solve for $\boldsymbol{u}_{\mathrm{f}}^{n+1}$. This final system will be diagonal and hence trivially inverted. In this process, $\boldsymbol{u}_{\mathrm{s}}^{n+1}$ for the solid porous materials is obtained with an implicit solve, ensuring that the timestep size is not restricted by explicit integration. We now elaborate on each of these three steps.

4.2.1 Pressure solve. We start with the explicit discretization of (1a), for which the $h^{2} \mathbf{H}$ term in front of $\boldsymbol{u}_{\mathrm{s}}^{n+1}$ in (22) vanishes. Then, the linear system consisting of (21), (23), and the explicit counterpart of (1a) can be written as (refer to section 1 of the supplemental material for the derivation)

$$
\begin{gathered}
{\left[\begin{array}{ccc}
\mathbf{D}_{\mathrm{s}} & 0 & h\left(\mathbf{V}_{\mathrm{s}}+\mathbf{V}_{\mathrm{f}} \mathbf{P}\right) \mathrm{G} \\
0 & \mathbf{D}_{\mathrm{f}} & h\left(\mathbf{V}_{\mathrm{f}}+\mathbf{V}_{\mathrm{s}} \mathbf{Q}\right) \mathrm{G} \\
h \mathrm{G}^{T}[\phi] & h \mathrm{G}^{T}[1-\phi] & 0
\end{array}\right]\left[\begin{array}{l}
\boldsymbol{u}_{\mathrm{s}}^{n+1} \\
\boldsymbol{u}_{\mathrm{f}}^{n+1} \\
\boldsymbol{p}^{n+1}
\end{array}\right]} \\
= \\
=\left[\begin{array}{c}
h f_{\mathrm{s}}+\mathbf{M}_{\mathrm{s}} \boldsymbol{u}_{\mathrm{s}}^{n}+\mathbf{P}\left(\mathbf{M}_{\mathrm{f}} \boldsymbol{u}_{\mathrm{f}}^{n}+h f_{\mathrm{f}}\right) \\
h f_{\mathrm{f}}+\mathbf{M}_{\mathrm{f}} \boldsymbol{u}_{\mathrm{f}}^{n}+\mathbf{Q}\left(\mathbf{M}_{\mathrm{s}} \boldsymbol{u}_{\mathrm{s}}^{n}+h f_{\mathrm{s}}\right) \\
0
\end{array}\right],
\end{gathered}
$$

where the matrices $P, Q, D_{s}$, and $D_{f}$ are

$$
\begin{aligned}
\mathbf{P} & =\left(\mathbf{M}_{\mathrm{f}}+h[\mathbf{C}] \mathbf{V}_{c}\right)^{-1} h[\mathbf{C}] \mathbf{V}_{c}, \\
\mathbf{Q} & =\left(\mathbf{M}_{\mathrm{s}}+h[\mathbf{C}] \mathbf{V}_{c}\right)^{-1} h[\mathbf{C}] \mathbf{V}_{c}, \\
\mathbf{D}_{\mathrm{s}} & =\mathbf{M}_{\mathrm{s}}+\mathbf{P M}_{\mathrm{f}} \text {, and } \mathbf{D}_{\mathrm{f}}=\mathbf{M}_{\mathrm{f}}+\mathbf{Q} \mathbf{M}_{\mathrm{s}} .
\end{aligned}
$$

Recall that [C] is a tridiagonal matrix. Let $\tilde{\mathbf{C}}$ denote one of its $3 \times 3$ diagonal subblocks defined by (16). Its off-diagonal element $\tilde{\mathrm{C}}_{i j}$ depicts the drag force along the axis $i$ induced by the liquid-solid velocity difference along a different axis $j$. The cross-axis terms in the drag tensor are responsible for rotational and shear effects, which can be assumed negligible under moderate Reynolds number [Bagchi and Balachandar 2002]. We therefore lump the off-diagonal elements of [C] into its diagonal elements, turning [C] into a fully diagonal matrix. This approximation can also be justified from a numerical point of view. When the drag force is large (e.g., for fast liquid flows wherein the Reynolds number is high), [C], without lumping, dominates over $\mathbf{M}_{\mathrm{f}}$ and $\mathbf{M}_{\mathbf{s}}$. Thus, $\mathbf{P}$ and $\mathbf{Q}$ in (25) are both nearly identity matrices, and $\mathbf{D}_{\mathrm{s}}$ and $\mathbf{D}_{\mathrm{f}}$ are nearly diagonal; lumping simply [C] approximates $\mathbf{D}_{\mathrm{s}}$ and $\mathbf{D}_{\mathrm{f}}$ as fully diagonal. On the other hand, when the drag force is very small, [C] approaches zero. Then, $\mathbf{P}$ and $\mathbf{Q}$ are close to zero, and $D_{s}$ and $D_{f}$ remain almost diagonal, so lumping [C] to be diagonal matrix is again a reasonable approximation.

With $D_{s}$ and $D_{f}$ being diagonal, the first two equations of (24) allow us to easily express $\boldsymbol{u}_{\mathrm{s}}^{n+1}$ and $\boldsymbol{u}_{\mathrm{f}}^{n+1}$ with respect to $\boldsymbol{p}^{n+1}$. After substituting this expression into the third equation of (24), we obtain a system of equations with respect to $\boldsymbol{p}^{n+1}$,

$$
\begin{array}{r}
h \mathrm{G}^{T}\left[[1-\phi] \mathbf{D}_{\mathrm{f}}^{-1}\left(\mathbf{V}_{\mathrm{f}}+\mathbf{V}_{\mathrm{s}} \mathbf{Q}\right)+[\phi] \mathbf{D}_{\mathrm{s}}^{-1}\left(\mathbf{V}_{\mathrm{s}}+\mathbf{V}_{\mathrm{f}} \mathbf{P}\right)\right] \mathrm{G} \boldsymbol{p}^{n+1} \\
=\mathrm{G}^{T}\left[\boldsymbol{\Phi}_{\mathrm{fs}}\left(\boldsymbol{u}_{\mathrm{f}}^{n}+\mathbf{M}_{\mathrm{f}}^{-1} f_{\mathrm{f}}\right)+\boldsymbol{\Phi}_{\mathrm{sf}}\left(\boldsymbol{u}_{\mathrm{s}}^{n}+\mathbf{M}_{\mathrm{s}}^{-1} f_{\mathrm{s}}\right)\right],
\end{array}
$$

where the matrices $\Phi_{\mathrm{fs}}$ and $\Phi_{\mathrm{sf}}$ have the following forms,

$$
\begin{aligned}
& \boldsymbol{\Phi}_{\mathrm{fs}}=[1-\phi] \mathbf{D}_{\mathrm{f}}^{-1} \mathbf{M}_{\mathrm{f}}+[\phi] \mathbf{D}_{\mathrm{s}}^{-1} \mathbf{M}_{\mathrm{f}} \mathbf{P}, \\
& \boldsymbol{\Phi}_{\mathrm{sf}}=[\phi] \mathbf{D}_{\mathrm{s}}^{-1} \mathbf{M}_{\mathrm{s}}+[1-\phi] \mathbf{D}_{\mathrm{f}}^{-1} \mathbf{M}_{\mathrm{s}} \mathbf{Q} .
\end{aligned}
$$

Equation (26) is analogous to the pressure projection step in standard fluid simulation, but for solid-liquid mixtures.

4.2.2 Solid velocity solve. After obtaining $\boldsymbol{p}^{n+1}$, we are ready to solve for $\boldsymbol{u}_{\mathrm{s}}^{n+1}$. Because of the high stretch stiffness of cloth and yarn, we adopt the implicit discretization in (22). Then, the $h^{2} \mathbf{H}$ term multiplying $\boldsymbol{u}_{\mathrm{s}}^{n+1}$ in (22) will appear in the first row of equations in (24): the first subblock $\mathbf{D}_{\mathrm{s}}$ becomes $\mathbf{D}_{\mathrm{s}}+h^{2} \mathbf{H}$. We obtain a system of equations with respect to $\boldsymbol{u}_{\mathrm{s}}^{n+1}$ :

$$
\begin{aligned}
&\left(\mathrm{D}_{\mathrm{s}}+h^{2} \mathbf{H}\right) \boldsymbol{u}_{s}^{n+1}=-h\left(\mathrm{~V}_{\mathrm{s}}+\right.\left.\mathrm{V}_{\mathrm{f}} \mathbf{P}\right) \mathrm{G} \boldsymbol{p}^{n+1}+ \\
& h f_{\mathrm{s}}+\mathbf{M}_{\mathrm{s}} \boldsymbol{u}_{\mathrm{s}}^{n}+\mathbf{P}\left(\mathbf{M}_{\mathrm{f}} \boldsymbol{u}_{\mathrm{f}}^{n}+h f_{\mathrm{f}}\right),
\end{aligned}
$$

where the pressure $\boldsymbol{p}^{n+1}$ is already known at this point. On the left-hand side, $\mathbf{D}_{\mathrm{s}}+h^{2} \mathbf{H}$ is a symmetric positive definite matrix. We then solve this system with a matrix-free conjugate-residual solver preconditioned with $\mathrm{D}_{\mathrm{s}}^{-1}$.

4.2.3 Fluid velocity solve. Lastly, we substitute $\boldsymbol{p}^{n+1}$ and $\boldsymbol{u}_{\mathrm{s}}^{n+1}$ into the second line of (24) to solve for $\boldsymbol{u}_{\mathrm{f}}^{n+1}$. As the matrix $\mathbf{D}_{\mathrm{s}}$ multiplying $\boldsymbol{u}_{\mathrm{f}}^{n+1}$ is diagonal, this equation is trivially solved, where

$$
\begin{aligned}
& \boldsymbol{u}_{\mathrm{f}}^{n+1}=\mathrm{D}_{\mathrm{f}}^{-1}\left[-h\left(\mathrm{~V}_{\mathrm{f}}+\mathrm{V}_{\mathrm{s}} \mathrm{Q}\right) \mathrm{G} \boldsymbol{p}^{n+1}+\right. \\
& \left.h f_{\mathrm{f}}+\mathbf{M}_{\mathrm{f}} \boldsymbol{u}_{\mathrm{f}}^{n}+\mathrm{Q}\left(\mathbf{M}_{\mathrm{s}} \boldsymbol{u}_{\mathrm{s}}^{n}+h f_{\mathrm{s}}\right)\right] .
\end{aligned}
$$

Remark. While we require implicit integration for stability of the fabric, we observed that within a single time step, the explicit and implicit methods produce similar fabric motion, especially when the time step is not too large. Therefore, we choose a semi-implicit approach in exchange for computational performance: by explicitly integrating velocity for the pressure solve, and implicitly integrating the fabric velocity after the pressure solve, we reduce the large, unsymmetric linear system to three smaller symmetric and positive definite systems which are much easier to solve.

When solving for the liquid velocity, we may either insert the pressure and solid velocity into (23) or only insert the pressure into the second line of (24). We tested both options. For the former choice, we observed an average of $\sim 3 \%$ difference in the divergence in all of our examples using the time step given in Table 1, while for the latter choice we observed an average difference in the divergence an order of magnitude smaller. The intuition is that in the former choice an additional Jacobian matrix $h^{2} \mathbf{H}$ is added to the divisor when solving for the matrix $Q$ in (25), which further increases the mismatch between the solved pressure and the divergence of liquid velocity. Hence we choose the latter solution.

We also found that the difference has approximately linear growth with respect to time step and the viscosity of the liquid. In practice, for liquid up to a moderate viscosity coefficient (e.g., olive oil), we did not observe any visual artifacts due to the difference. Nevertheless for high viscosity liquid (e.g., honey) there is indeed some instability due to the mismatch between the explicitly integrated solid velocity used by the drag force, and the actual implicitly integrated solid 
velocity. A method with a strict guarantee of incompressibility and which can handle high viscosity liquid requires future investigation.

4.2.4 Implementation details. In the aforementioned three steps, we need to construct $\mathbf{M}_{\mathrm{s}}, \mathbf{V}_{\mathrm{c}}, \mathbf{V}_{\mathrm{s}}, \mathbf{V}_{\mathrm{f}}$, and $\mathbf{M}_{\mathrm{f}}$ for the face centers of the MAC grid. For $\mathbf{M}_{\mathrm{s}}, \mathbf{V}_{\mathrm{c}}, \mathbf{V}_{\mathrm{s}}$, and $\mathbf{V}_{\mathrm{f}}$, we first compute the corresponding quantities on cloth and yarn vertices. For example, the liquid volume $V_{f}$ at a vertex is computed with $V_{\mathrm{V}}(1-\phi) S_{\mathrm{r}}$, where $\phi$ is the solid volume fraction at that vertex, and $S_{\mathrm{r}}$ is its saturation We then distribute the quantities from the vertices to face centers, using the kernel functions defined in the APIC method [Jiang et al. 2015]. Similarly, for constructing $\mathbf{M}_{\mathrm{f}}$, we compute the liquid mass $\rho_{\mathrm{f}} V_{\mathrm{f}, p}$ on each liquid particle, and distribute it to the face centers.

Force computation. In the discretized equations (22) and (23), the forces are computed as

$$
f_{\mathrm{s}}=\mathrm{V}_{\mathrm{s}} \nabla \cdot\left[\left(1-S_{\mathrm{r}}\right) \mathrm{P}_{\mathrm{c}}\right]+f_{\mathrm{F}} \text { and } f_{\mathrm{f}}=\mathrm{V}_{\mathrm{f}} \nabla \cdot\left[\left(1-S_{\mathrm{r}}\right) \mathrm{P}_{\mathrm{c}}\right]+\mathbf{M}_{\mathrm{f}} \boldsymbol{g} \text {, }
$$

where $\mathbf{P}_{\mathrm{C}}$, as defined in (11), is the suction tensor computed using the quantities stored on grid face centers, and $S_{\mathrm{r}}$ is the solid vertex saturation distributed on the grid. Hence the first terms of both $f_{\mathrm{s}}$ and $f_{\mathrm{f}}$ are directly evaluated on grid face centers, and $\mathbf{M}_{\mathrm{f}} \boldsymbol{g}$ is the liquid's gravity force evaluated on the grid as well. On the other hand, $f_{\mathrm{F}}$ are forces applied on solid vertices, including the internal elastic forces, collision and frictional forces, and gravity forces. These are evaluated at individual solid vertices and distributed to grid faces using the APIC method. We adopt existing models to compute these forces. In particular, the cloth internal forces are computed using the discrete shell model [Grinspun et al. 2003], the internal forces of yarn follow the discrete elastic rod model [Bergou et al. 2010], and the collision and frictional forces are computed following Jiang et al. [2017].

We highlight one detail related to the distribution of yarn torques using APIC. The discrete viscous thread model uses a scalar $t_{\mathrm{V}}$ at each yarn vertex to indicate the strength of torque with respect to the tangential direction $\boldsymbol{d}_{\mathrm{V}}$ of the yarn. In order to distribute the torque to a background grid face $i$, we convert the scalar into a vector $t_{\mathrm{V}} d_{\mathrm{V}} \times \nabla w_{i, \mathrm{~V}}$ (see derivation in section 2 of the supplemental material) before adding it to the grid face $i$. Here $w_{i, \mathrm{~V}}$ is the kernel function between grid face $i$ and solid vertex $\mathbf{V}$.

facobian matrix computation. The matrix $\mathbf{H}$ in (22) and (23) is the Jacobian of $f_{\mathrm{s}}$ distributed on the grid. This matrix emerges when we integrate the force terms implicitly. Because the stiffest force terms in $f_{\mathrm{s}}$ are the internal elastic forces, we compute their contributions to the Jacobian matrix, and ignore the contributions from collision and frictional forces, instead integrating them explicitly. We compute the Jacobian matrix $\mathbf{H}_{\mathrm{V}}$ of the elastic forces at each solid vertex $\mathrm{V}$, and add its contribution to the grid face $i$ using

$$
\mathbf{H}_{i, \mathrm{~V}}=\mathbf{W}_{i \rightarrow \mathrm{V}}^{T} \mathrm{H}_{\mathrm{V}} \mathbf{W}_{i \rightarrow \mathrm{V}},
$$

where $\mathbf{W}_{i \rightarrow \mathrm{V}}$ is the weight that distributes a force vector from grid face $i$ to solid vertex $\mathrm{V}$ in the augmented MPM method, as defined by Stomakhin et al. [2014].

Cohesion force between cloth. When two pieces of wet cloth are in close proximity, the surface tension of the liquid between introduces cohesion forces. Accurately computing surface tension requires the reconstruction of detailed liquid surface shapes between the cloth, which in turn demands an extremely high grid resolution. Even for a moderate size piece of cloth, computing this effect through brute force is intractable. In this work, we use a simple model to approximate cohesion forces at cloth vertices, and use the APIC method again to distribute the forces to grid nodes. We describe our model in section 3 of the supplemental material, while leaving a full investigation of this surface tension-induced effect to future research. For the cohesion between yarns, we took the model by Fei et al. [2017] to compute the force.

\section{RESULTS}

We divide our results into two classes: $i$ ) a group of didactic cases designed to validate individual components of our framework, and ii) a set of more general scenarios of liquid interaction with cloth and yarn that demonstrate the diversity of practical effects that can be achieved by our system. Details of our surface reconstruction and rendering method can be found in section 4 of the supplemental material. A summary and discussion of the physics parameters used throughout this paper can be found in section 5 of the supplemental material.

\subsection{Didactic Examples}

Ring Test. A classic experiment in the textiles industry is a ring test [Patnaik et al. 2006], where a controlled volume of liquid is released onto the center of a piece of cloth. We compare our simulation with a physical experiment in Figure 3. When the liquid touches the cloth, wicking can be observed in both the physical experiment and our simulation. Although our numerical experiment does not quite reproduce the noisy details of the real-world surface, the liquid in both the experiment and our simulation yielded visually and qualitatively consistent wicking behaviors.

Drag Forces. The nonlinearity of drag forces has a significant impact on the look of real liquid-cloth interactions. Figure 6 presents a comparison between nonlinear and linear drag force models. The most obviously distinct visual phenomenon that can be seen in the nonlinear case is the formation of "kinks" around regions where the relative velocity between the cloth and liquid is large; the cloth has dragged the liquid along with it to create this characteristic shape. This phenomenon cannot be readily observed with the linear drag force. The same figure illustrates this "kink" effect in a real experiment in which large relative velocities are induced by pulling a cloth rapidly out of liquid.

Buoyancy Forces. In Figure 4, we highlight the importance of the pressure gradient, using fabrics with differing mass densities. The leftmost has density $\rho_{s}=0.25 \mathrm{~g} / \mathrm{cm}^{3}$, which is lower than water's; the middle fabric has the same density $\rho_{s}=1.0 \mathrm{~g} / \mathrm{cm}^{3}$ as water (i.e., neutrally buoyant); and the rightmost has density $\rho_{s}=4.0 \mathrm{~g} / \mathrm{cm}^{3}$, which is higher than water's. With the correct pressure gradient applied to the fabrics, as expected, the left one rises to the water surface; the middle one drifts along with the fluid water; and the right one sinks quickly to the bottom. By contrast, if the pressure gradient is neglected, the fabrics sink and come to rest at the bottom, regardless of their mass densities. 


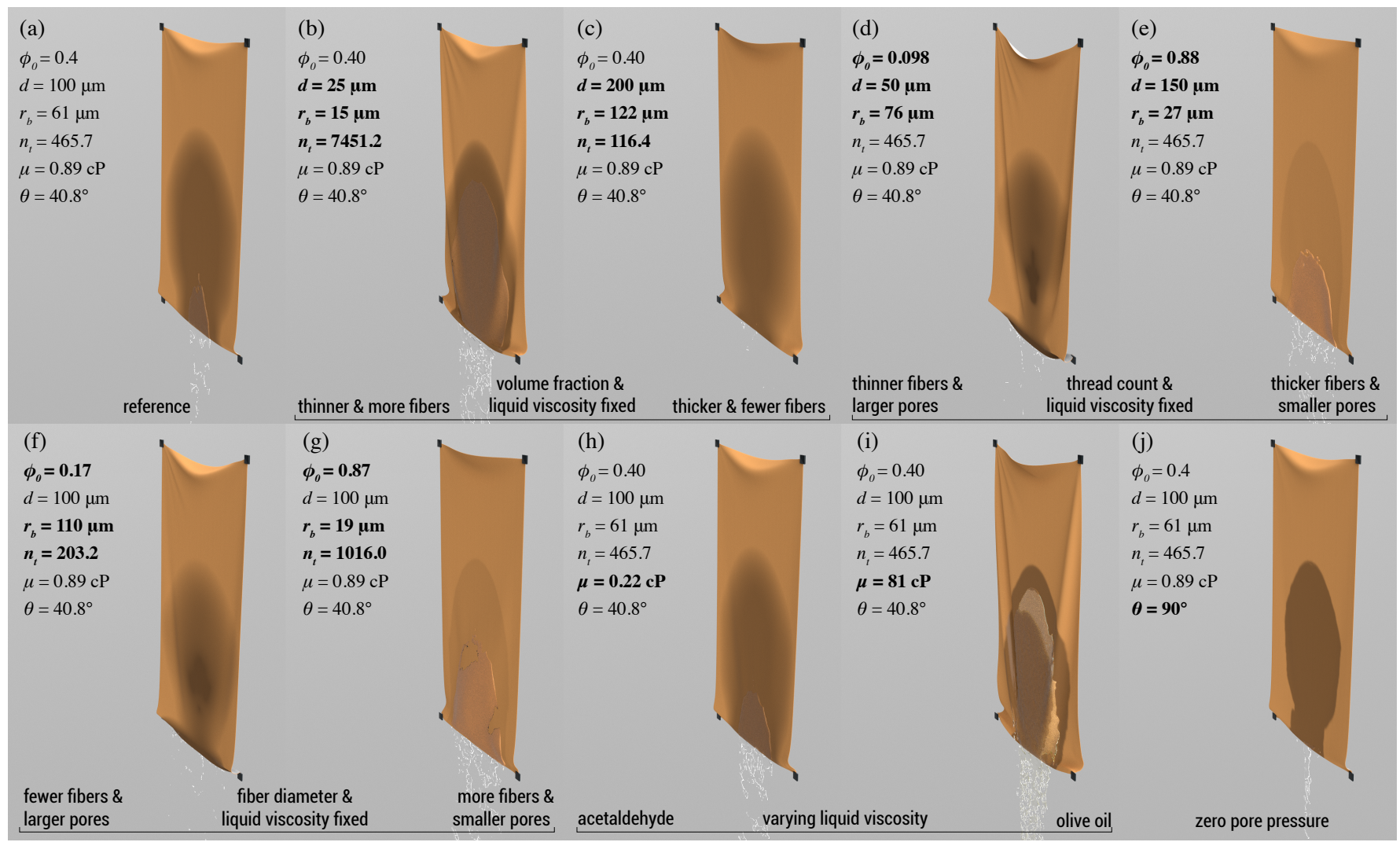

Fig. 10. Comparison for different sets of fabric and liquid parameters. Parameters different from the reference's are highlighted with bold text. The fabric parameters include rest solid fraction $\phi_{0}$ (unitless), fiber diameter $d$ (micrometers), capillary radius $r_{b}$ (micrometers), and thread count per inch $n_{t}$ (unitless), where any two of them can be determined by the other two. The liquid parameters include viscosity $\mu$ (centipoise) and contact angle $\phi$ (degrees).

Various Parameters. Different fabric and liquid parameters can also drastically alter the look of cloth-liquid interaction [Das et al. 2008]: permeability decreases quadratically with fiber diameter and nonlinearly with volume fraction (Equation (13)); while the pore pressure increases with volume fraction and decreases with contact angle (Equation (9)). In Figure 10 we compare simulation with different sets of parameters varied from reference (Figure 10a). All the simulations are done with the same initial geometries, and the screenshots are captured at 4.0 seconds. The expected effects are recovered in our numerical experiments. In Figure $10 \mathrm{~b}$ and 10c, as we adjust the fiber diameter $d$, we simultaneously hold the rest solid fraction $\phi_{0}$ constant by appropriately adjusting the thread count $n_{t}$ and capillary radius $r_{b}$ to compensate; the cloth with smaller $d$ is less readily penetrated by the liquid, the liquid attaches more readily to the cloth surface, and a shorter wicking distance is observed. In Figure $10 \mathrm{~d}$ and $10 \mathrm{e}$, as we adjust the fiber diameter $d$, we simultaneously hold the thread count $n_{t}$ constant; as $d$ and the rest solid fraction $\phi_{0}$ increased, the cloth is less easily penetrated by the liquid, also with less liquid retention inside the cloth. In Figure $10 \mathrm{f}$ and $10 \mathrm{~g}$, we change the thread count $n_{t}$, while holding the fiber diameter $d$ constant; as $n_{t}$ and the rest solid fraction $\phi_{0}$ increased, the cloth shows a similar behavior. We also compare between different liquid parameters. In Figure 10h and 10i, we demonstrate the different behavior of acetaldehyde and olive oil, where the former is less viscous and the latter is much more viscous than water: the cloth is less easily penetrated by olive oil, which also has a much shorter wicking distance. In Figure 10j, we demonstrate the effect where zero pore pressure is applied when the contact angle is exactly 90 degrees: there is then no wicking effect and the liquid is less attracted to the cloth surface.

\section{Impermeable Cloth. We} show that our method can also simulate liquid coupled with a pinned impermeable (non-porous) cloth, which corresponds to an infinitely small pore

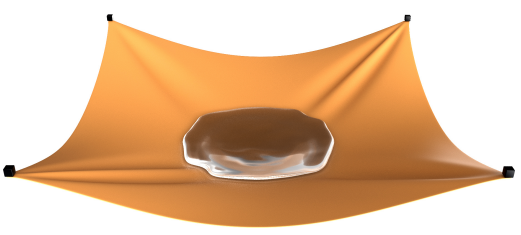
size and infinitely large drag force in our model. Since the drag force is implicitly integrated (equation 24, 28 and 29), our simulation is valid even when the drag tensor approaches infinity. Around the overlapped region, the liquid and solid share the same velocity, corresponding to a no-slip boundary condition.

\subsection{General Examples}

Splash on Cloth. Figure 11 demonstrates wetting, dragging, dripping, and wicking effects of liquid-cloth interaction. When the liquid has high velocity, it can penetrate through the cloth from one side to another, but as it is slowed down by viscous drag, it will attach 


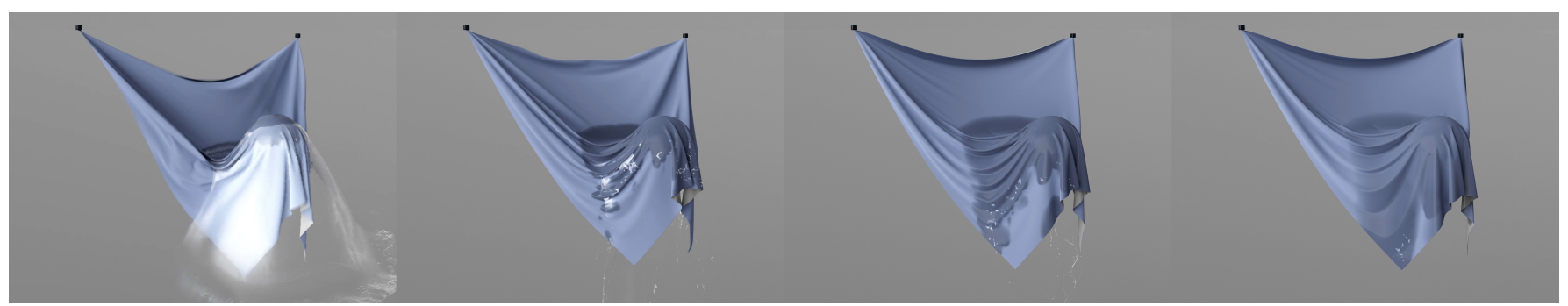

Fig. 11. A ball of water splashes on a mesh-based cloth.

to the cloth surface and start to slip. As more liquid attaches to the cloth, the cloth also starts drooping due to the added mass.

Splash on Yarns. Similarly, in Figure 12 we show that our model can handle yarn-based fabrics by dropping a ball of water on a piece of pinned handwoven fabric. Some of the liquid is captured by the fibers, while the majority of it flows through the pores and forms a liquid jet on the other side. The fabric is also noticeably tightened by the initial impact of the water ball.

Splash on Fuzzy. Beyond cloth and yarn, we show that our model can handle a scenario involving both kinds of structure: in Figure 13 we splash a ball of water onto a fuzzy cloth that has many short strands protruding from its surface. This cloth has a stiffer visual look than regular cloth, it absorbs more water, and the drag force is also stronger.

Tighten the Towel. Lastly, we show an example with more complicated dynamics in which the motion of a fuzzy cloth actively affects the flow of a liquid. Specifically, in Figure 14 we simulate the tightening of a towel. The towel is rapidly yanked out of water and tightened. As the towel twists, a sudden rush of liquid flows out of the towel. As time goes on, the flow of liquid leaving the towel steadily decreases to a trickle.

For both Tighten the Towel and Drag Forces we measured the total volume of liquid on the towel and in bulk form over the course of the simulation. The volume of the bulk liquid is calculated as the sum of the spherical volumes of liquid associated with each APIC particle, according to each particle's radius. The volume of the liquid on the towel is calculated as the sum of the liquid stored on the vertices. For each solid vertex the liquid volume is simply the saturation multied by the empty pore space. Figure 15, left, shows that the net increase of water on the mesh (blue curve) was always offset by the net decrease in bulk liquid (orange curve), yielding remarkably good conservation of total liquid volume (green curve).

\subsection{Performance Numbers}

In Table 1 we collected timing data to evaluate the computational cost of our method and its various components on our examples, using a workstation with four Intel Xeon E5-2687W CPUs with eight cores each running at $3.10 \mathrm{GHz}$. For the towel example we also provide a detailed breakdown in Figure 16. The most time consuming part is for the calculation of the forces, plasticity and interpolation kernel weights. Throughout the paper, we use a cell size of $\delta x=0.288 \mathrm{~cm}$, with an average distance between mesh vertices of $0.144 \mathrm{~cm}$. Since our grid is built only in a neighborhood
Table 1. Timings and storage statistics.

\begin{tabular}{r|ccccc}
\hline Examples & $\begin{array}{c}\text { s/step } \\
\text { (Avg.) }\end{array}$ & $\begin{array}{c}\text { \# particle } \\
(\mathrm{Max})\end{array}$ & \# element & $h(\mathrm{~s})$ & $\begin{array}{c}\text { Mem. } \\
(\text { Peak GB })\end{array}$ \\
\hline Drag Forces & 5.93 & $570 \mathrm{~K}$ & $8.54 \mathrm{~K}$ & $2 \times 10^{-4}$ & 5.41 \\
Buoyancy Forces & 6.02 & $742 \mathrm{~K}$ & $105 \mathrm{~K}$ & $2 \times 10^{-4}$ & 6.06 \\
Various Parameters & 1.88 & $199 \mathrm{~K}$ & $64.1 \mathrm{~K}$ & $2 \times 10^{-4}$ & 4.39 \\
Impermeable Cloth & 3.20 & $112 \mathrm{~K}$ & $71.8 \mathrm{~K}$ & $2 \times 10^{-4}$ & 2.44 \\
Splash on Cloth & 18.73 & $729 \mathrm{~K}$ & $336 \mathrm{~K}$ & $2 \times 10^{-4}$ & 18.35 \\
Splash on Yarns & 2.72 & $277 \mathrm{~K}$ & $79.6 \mathrm{~K}$ & $2 \times 10^{-4}$ & 6.19 \\
Splash on Fuzzy & 9.31 & $282 \mathrm{~K}$ & $123 \mathrm{~K}$ & $2 \times 10^{-4}$ & 5.27 \\
Tighten the Towel & 8.97 & $390 \mathrm{~K}$ & $71.2 \mathrm{~K}$ & $2 \times 10^{-4}$ & 4.90 \\
\hline
\end{tabular}

around the solid vertices and liquid particles, its size is temporally variant. The number of cells varies between 50 and 250 in the largest dimension for all of our examples.

\section{DISCUSSION AND LIMITATIONS}

We have presented a numerical model to animate liquid interactions with permeable cloth and yarn that is able to capture many key phenomena. We highlight below a few limitations imposed by our chosen assumptions, numerical methods, or experiments.

In Figure 3 we compared the diffusion simulated by our method with a laboratory experiment. To better match the laboratory result (Figure 3left), we experimented with using a manually specified volume fraction

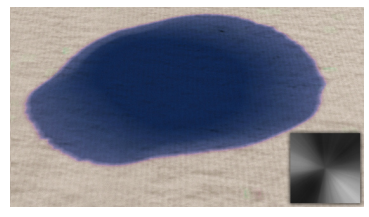
field on the textile (visualized in the bottom-right of the adjacent figure). While this leads to non-uniform diffusion closer to the laboratory result, we found very difficult to match perfectly. This is because there are other factors that would affect the diffusion, such as the spatially varying fiber radii that changes the pore pressure and the abrasion of the textile sample that produces irregular bumps on the surface. In future work it would be worth investigating how to model and incorporate these textile "defects" for more realistic simulation.

Our fiber model makes assumptions about the dominant axes of the pore structure, which places limitations on the fidelity of our pore pressure and drag forces for general microstructures; for example, yarn strands in the fuzzy cloth are assumed to attach perpendicularly to the cloth. For numerical efficiency, our drag model also relied on a lumping strategy that assumes shear and rotational effects are relatively unimportant.

Since the liquid bridge geometry that causes wet cloth sticking is difficult to model, we adopted a fairly simple cohesion approach. Of course, there are situations in which cohesion has a very meaningful influence on the dynamics: consider the manner in which wet clothes 


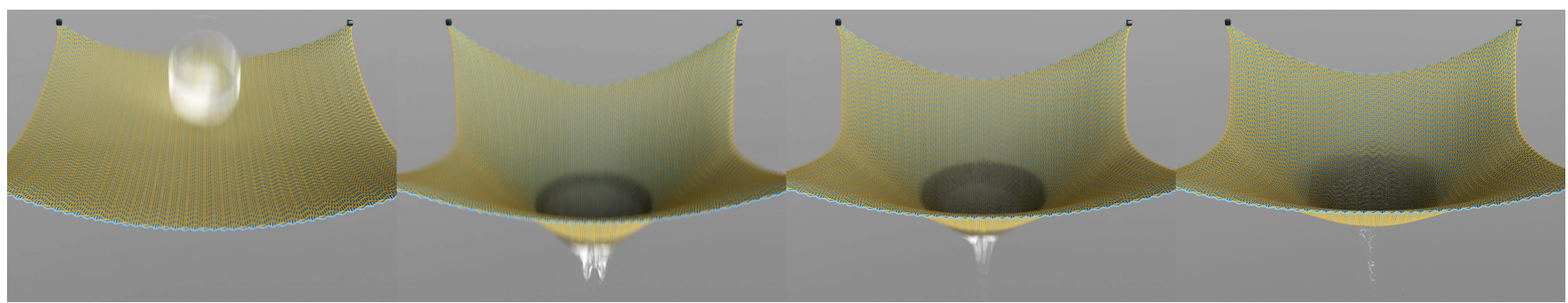

Fig. 12. A ball of water splashes on a yarn-based fabric.

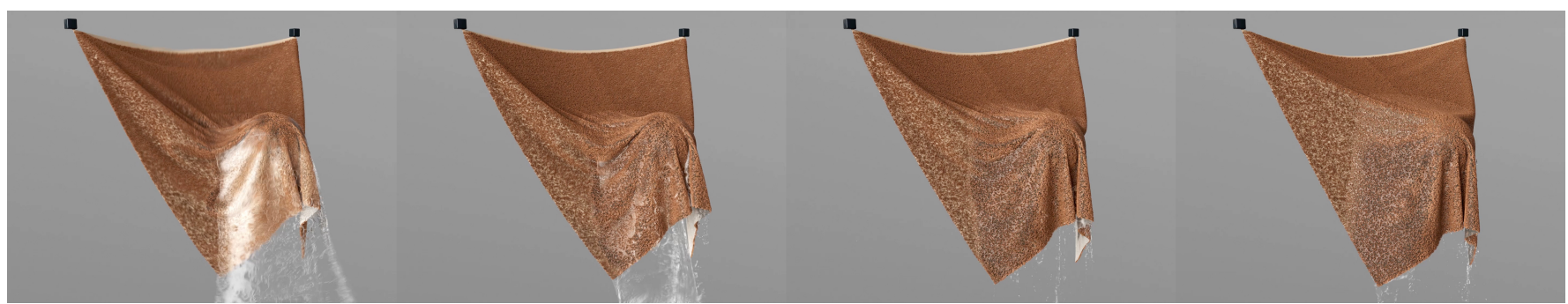

Fig. 13. A ball of water splashes on a fuzzy cloth.

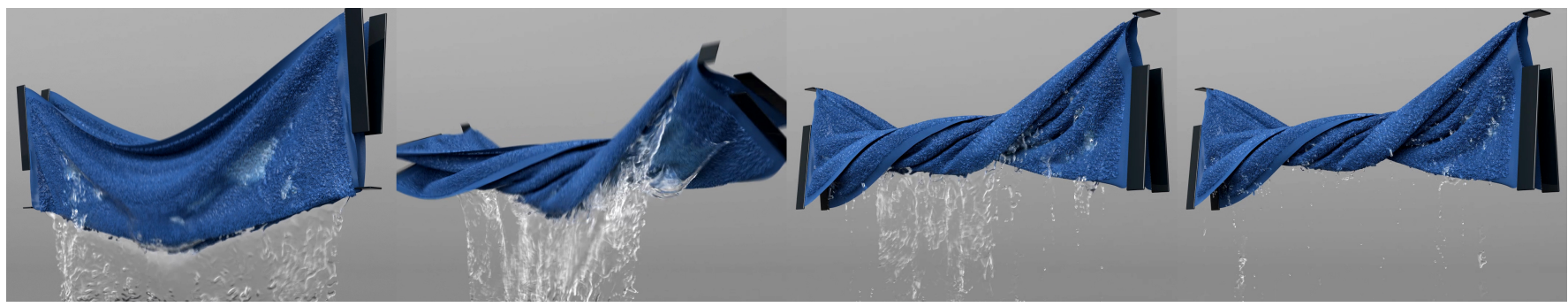

Fig. 14. A towel is pulled rapidly out of water and wrung out.
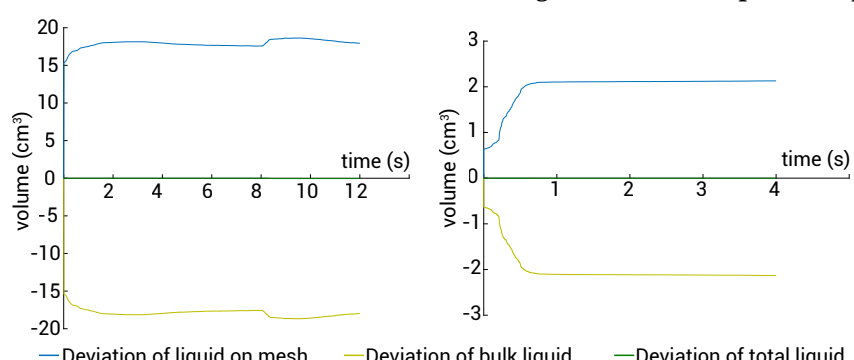

Fig. 15. Volume conservation is demonstrated by plotting the deviation of fluid volume in bulk form (orange), on the cloth (blue), and their total (green). Left: Tighten the Towel example. Right: Drag Forces example.

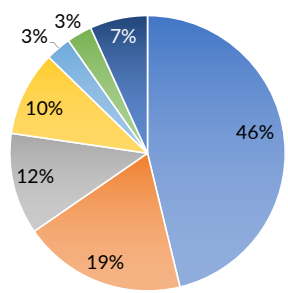

$$
\begin{aligned}
& \text { - Implicit Integration of Solid Velocity } \\
& \text { - Compute Force \& Kernel Weights } \\
& \text { = Solve Quasi-Static Equation } \\
& \text { = Build Sparse MAC Grid } \\
& \text { - Velocity Prediction } \\
& \text { - Pressure Projection } \\
& \text { - Others }
\end{aligned}
$$

Fig. 16. Performance breakdown for Tighten the Towel (Figure 14).

adhere to one's body. Relatedly, we did not include surface tension in the bulk fluid flow, though adding an explicitly integrated approach would likely be straightforward.
More fundamentally, our system relies on mixture and porous flow theories, which themselves entail a variety of both limitations along with benefits. Principally, they assume continuum models of the phenomena and their interactions, for example abstracting away real fine-scale geometry of individual droplets and pores. In both engineering and animation this extreme level of detail is often superfluous, though not universally. For example, in the ring test, it is likely that we might recover some of the differences from the physical experiment with a more faithful coarse-scale model of the specific fabric geometry we used; however, certain small-scale heterogeneities, wrinkles, etc. seem likely to remain beyond the reach of our scheme.

We adopted weak coupling through the drag force and do not enforce an exact matching of velocity at the interface. The liquid and solid are treated as a continuum mixture, and the drag force acts to pull the liquid velocity closer to that of the solid. The scale of the drag force depends on the solid permeability. As such, the solid velocity will only exactly match the liquid velocity in the limit of infinitely large drag force (corresponding to the scenario of an impermeable cloth). We indeed observed artifacts when the discretization is too coarse, which is a limitation of continuum modeling.

At present, our method relies on a relatively fine grid resolution to achieve realistic results: the fabric thickness is not even a full order of magnitude smaller than a grid cell. Ideally, one would prefer a large gap to reduce the significant cost of the volumetric 
solve, although ensuring seamless interactions under such coarse conditions appears non-trivial. In a related vein, strictly speaking our model is semi-implicit which implies a timestep restriction that could slow our results; a fully implicit solution remains an open question. However, in practice, the primary factor driving timestep selection was collision-handling, rather than internal dynamics. For the sake of efficiency, we also adopted a free surface model that avoids simulating the bulk air volume; this offers a faster simulation but sacrifices air-dependent effects such as (grid-scale) bubbles.

\section{ACKNOWLEDGMENTS}

This work was supported in part by the National Science Foundation under Grant Nos.: 1717178, 1319483, CAREER-1453101, the Natural Sciences and Engineering Research Council of Canada under Grant No. RGPIN-04360-2014, SoftBank Group, Pixar, and Adobe. We also thank Gilles Daviet and Peter Yichen Chen for insightful discussions, Chang Xiao, Henrique Maia and Oded Stein for their assistance, Xinxin Zhang for his AMGPCG pressure solver [2015], Robert Lane and Jorge Espinoza for their help on storage and computational devices, and Cristin Barghiel and Julie Lottering for their continued generous contribution from SideFX.

\section{REFERENCES}

Keita Abe, Kenichi Soga, and Samila Bandara. 2013. Material point method for coupled hydromechanical problems. Fournal of Geotechnical and Geoenvironmental Engineering 140, 3 (2013), 04013033.

KJ Ahn, JC Seferis, and JC Berg. 1991. Simultaneous measurements of permeability and capillary pressure of thermosetting matrices in woven fabric reinforcements Polymer Composites 12, 3 (1991), 146-152.

Ömer Akgiray and Ahmet M Saatç1. 2001. A new look at filter backwash hydraulics. Water Science and Technology: Water Supply 1, 2 (2001), 65-72.

Nadir Akinci, Jens Cornelis, Gizem Akinci, and Matthias Teschner. 2013. Coupling elastic solids with smoothed particle hydrodynamics fluids. Computer Animation and Virtual Worlds 24, 3-4 (2013), 195-203.

SC Amico and C Lekakou. 2002. Axial impregnation of a fiber bundle. Part 2: theoretical analysis. Polymer composites 23, 2 (2002), 264-273.

T B Anderson and Roy Jackson. 1967. Fluid mechanical description of fluidized beds Equations of motion. Industrial \& Engineering Chemistry Fundamentals 6, 4 (1967), 527-539.

Omri Azencot, Orestis Vantzos, Max Wardetzky, Martin Rumpf, and Mirela BenChen. 2015. Functional thin films on surfaces. In Proceedings of the 14th ACM SIGGRAPH/Eurographics Symposium on Computer Animation. ACM, 137-146.

Vinicius C Azevedo, Christopher Batty, and Manuel M Oliveira. 2016. Preserving geometry and topology for fluid flows with thin obstacles and narrow gaps. ACM Transactions on Graphics (TOG) 35, 4 (2016), 97.

P Bagchi and S Balachandar. 2002. Effect of free rotation on the motion of a solid sphere in linear shear flow at moderate Re. Physics of Fluids 14, 8 (2002), 2719-2737.

Samila Bandara and Kenichi Soga. 2015. Coupling of soil deformation and pore fluid flow using material point method. Computers and geotechnics 63 (2015), 199-214.

Jacob Bear. 2013. Dynamics of fluids in porous media. Courier Corporation.

A Bedford and D S Drumheller. 1983. Theories of immiscible and structured mixtures. International fournal of Engineering Science 21, 8 (1983), 863-960.

Miklós Bergou, Basile Audoly, Etienne Vouga, Max Wardetzky, and Eitan Grinspun. 2010. Discrete viscous threads. ACM Transactions on Graphics (TOG) 29, 4 (2010), 116.

José Bico, Étienne Reyssat, and Benoît Roman. 2018. Elastocapillarity: When Surface Tension Deforms Elastic Solids. Annual Review of Fluid Mechanics 50, 1 (2018), 629-659.

Maurice A Biot. 1941. General theory of three-dimensional consolidation. Fournal of applied physics 12, 2 (1941), 155-164.

Javier Bonet and Richard D Wood. 1997. Nonlinear continuum mechanics for finite element analysis. Cambridge university press.

Ronaldo I Borja. 2006. On the mechanical energy and effective stress in saturated and unsaturated porous continua. International fournal of Solids and Structures 43, 6 (2006), 1764-1786.

Mario Botsch, Leif Kobbelt, Mark Pauly, Pierre Alliez, and Bruno Lévy. 2010. Polygon mesh processing. CRC press.
Robert Bridson. 2015. Fluid simulation for computer graphics. CRC Press

Royal Harvard Brooks and Arthur Thomas Corey. 1964. Hydraulic properties of porous media. Hydrology papers (Colorado State University) 3 (1964).

Richard L Burden and J Douglas Faires. 1985. 2.2 Fixed-Point Iteration. Numerical Analysis (3rd ed.). PWS Publishers. ISBN 0-87150-857-5 (1985).

Philip Crosbie Carman. 1937. Fluid flow through granular beds. Transactions-Institution of Chemical Engineeres 15 (1937), 150-166.

Yujun Chen, Nadia Magnenat Thalmann, and Brian Foster Allen. 2012. Physical simulation of wet clothing for virtual humans. The Visual Computer 28, 6-8 (2012), 765-774.

Nelson S.-H. Chu and Chiew-Lan Tai. 2005. MoXi: Real-time Ink Dispersion in Absorbent Paper. ACM Transactions on Graphics (TOG) 24, 3 (July 2005), 504-511.

S Chwastiak. 1973. A wicking method for measuring wetting properties of carbon yarns. Fournal of Colloid and Interface Science 42, 2 (1973), 298-309.

Gabriel Cirio, Jorge Lopez-Moreno, David Miraut, and Miguel A Otaduy. 2014. Yarnlevel simulation of woven cloth. ACM Transactions on Graphics (TOG) 33, 6 (2014), 207.

Cassidy J. Curtis, Sean E. Anderson, Joshua E. Seims, Kurt W. Fleischer, and David H. Salesin. 1997. Computer-generated Watercolor. In Proceedings of the 24th Annual Conference on Computer Graphics and Interactive Techniques (SIGGRAPH '97). ACM Press/Addison-Wesley Publishing Co., New York, NY, USA, 421-430.

Trong Dang-Vu and Jan Hupka. 2005. Characterization of porous materials by capillary rise method. Physicochemical problems of mineral processing 39 (2005), 47-65.

Henry Philibert Gaspard Darcy. 1856. Dètermination des lois d'ècoulement de l'eau à travers le sable.

Brojeswari Das, A Das, VK Kothari, R Fanguiero, and M Araujo. 2007. Moisture transmission through textiles. Part II: evaluation methods and mathematical modeling. Autex Res 7 7, 3 (2007), 194-216.

Brojeswari Das, A Das, VK Kothari, R Fanguiero, and M De Araújo. 2008. Effect of fibre diameter and cross-sectional shape on moisture transmission through fabrics. Fibers and Polymers 9, 2 (2008), 225-231.

Gilles Daviet and Florence Bertails-Descoubes. 2017. Simulation of Drucker-Prager granular flows inside Newtonian fluids. (Feb. 2017). working paper or preprint.

Reint De Boer. 2012. Theory of porous media: highlights in historical development and current state. Springer Science \& Business Media.

M de Saint-Venant. 1856. Mémoire sur la torsion des prismes: avec des considérations sur leur flexion ainsi que sur l'équilibre intérieur des solides élastiques en général et des formules pratiques pour le calcul de leur résistance à divers efforts s'exerçant simultanément. Imprimerie nationale.

Sabri Ergun. 1952. Fluid flow through packed columns. Chem. Eng. Prog. 48 (1952), 89-94.

Yun (Raymond) Fei, Henrique Teles Maia, Christopher Batty, Changxi Zheng, and Eitan Grinspun. 2017. A Multi-scale Model for Simulating Liquid-hair Interactions. ACM Transactions on Graphics (TOG) 36, 4, Article 56 (July 2017), 17 pages.

Adolf Fick. 1855. Ueber diffusion. Annalen der Physik 170, 1 (1855), 59-86.

Paul Fillunger. 1913. Der auftrieb in talsperren. Osterr. Wochenschrift fur den offentl. Baudienst 19, 32 (1913), 532-555.

PH Forchheimer. 1901. Wasserbewegung durch boden. Zeitz. Ver. Duetch Ing. 45 (1901), 1782-1788.

Eitan Grinspun, Anil N. Hirani, Mathieu Desbrun, and Peter Schröder. 2003. Discrete Shells. In Proceedings of the 2003 ACM SIGGRAPH/Eurographics Symposium on Computer Animation (SCA '03). Eurographics Association, Aire-la-Ville, Switzerland, Switzerland, 62-67.

Eran Guendelman, Andrew Selle, Frank Losasso, and Ronald Fedkiw. 2005. Coupling water and smoke to thin deformable and rigid shells. ACM Transactions on Graphics (TOG) 24, 3 (2005), 973-981.

Henry Selby Hele-Shaw. 1898. The flow of water. Nature 58, 1489 (1898), 33-36

Markus Huber, Bernhard Eberhardt, and Daniel Weiskopf. 2015. Boundary handling at cloth-fluid contact. Computer Graphics Forum 34, 1 (2015), 14-25.

Markus Huber, Simon Pabst, and Wolfgang Straßer. 2011. Wet cloth simulation. In ACM SIGGRAPH 2011 Posters. ACM, 10.

Markus Ihmsen, Jens Cornelis, Barbara Solenthaler, Christopher Horvath, and Matthias Teschner. 2014. Implicit incompressible SPH. IEEE Transactions on Visualization and Computer Graphics 20, 3 (2014), 426-435.

Chenfanfu Jiang, Theodore Gast, and Joseph Teran. 2017. Anisotropic elastoplasticity for cloth, knit and hair frictional contact. ACM Transactions on Graphics (TOG) 36, 4 (2017), 152.

Chenfanfu Jiang, Craig Schroeder, Andrew Selle, Joseph Teran, and Alexey Stomakhin 2015. The affine particle-in-cell method. ACM Transactions on Graphics (TOG) 34, 4 (2015), 51.

Jonathan M. Kaldor, Doug L. James, and Steve Marschner. 2008. Simulating Knitted Cloth at the Yarn Level. ACM Transactions on Graphics (TOG) 27, 3, Article 65 (Aug. 2008), 9 pages.

Jonathan M. Kaldor, Doug L. James, and Steve Marschner. 2010. Efficient Yarn-based Cloth with Adaptive Contact Linearization. ACM Transactions on Graphics (TOG) 29, 4, Article 105 (July 2010), 10 pages. 
HS Kim. 2003. In-plane liquid distribution in nonwoven fabrics: Part 2-simulation. Int Nonwoven 7. 12 (2003), 29-33

Erik Kissa. 1996. Wetting and wicking. Textile Research fournal 66, 10 (1996), 660-668.

Mark Landeryou, Ian Eames, and A Cottenden. 2005. Infiltration into inclined fibrous sheets. Fournal of Fluid Mechanics 529 (2005), 173-193.

C Lekakou and MG Bader. 1998. Mathematical modelling of macro-and microinfiltration in resin transfer moulding (RTM). Composites Part A: Applied Science and Manufacturing 29, 1-2 (1998), 29-37.

Toon Lenaerts, Bart Adams, and Philip Dutré. 2008. Porous flow in particle-based fluid simulations. ACM Transactions on Graphics (TOG) 27, 3 (2008), 49.

Wei-Chin Lin. 2014. Coupling Hair with Smoothed Particle Hydrodynamics Fluids In Workshop on Virtual Reality Interaction and Physical Simulation, Jan Bender Christian Duriez, Fabrice Jaillet, and Gabriel Zachmann (Eds.). The Eurographics Association.

Wei-Chin Lin. 2015. Boundary handling and porous flow for fluid-hair interactions. Computers \& Graphics 52 (2015), 33-42.

Lin Lou, Feng Ji, and Yiping Qiu. 2015. Simulating adhesion of wet fabrics to water: surface tension-based theoretical model and experimental verification. Textile Research fournal 85, 19 (2015), 1987-1998.

Lin Lou, Yiping Qiu, Feng Ji, and Xiaohang Zhu. 2018. The influence of surface hydrophilicity on the adhesion properties of wet fabrics or films to water. Textile Research fournal 88, 1 (2018), 108-117.

Lin Lou, Jianfei Xie, Feng Ji, Yiping Qiu, Xiaohang Zhu, and Jing Xu. 2017. Simulating adhesion of wet fabrics to water: Gravity of liquid bridge-based theoretical model and experimental verification. Textile Research fournal 87, 7 (2017), 769-779.

R Lucas. 1918. Rate of capillary ascension of liquids. Kolloid Z 23, 15 (1918), 15-22.

Hernán A Makse, David L Johnson, and Lawrence M Schwartz. 2000. Packing of compressible granular materials. Physical review letters 84, 18 (2000), 4160.

Reza Masoodi and Krishna M Pillai. 2012a. A general formula for capillary suctionpressure in porous media. Journal of Porous Media 15, 8 (2012).

Reza Masoodi and Krishna M Pillai. 2012b. Wicking in porous materials: traditional and modern modeling approaches. CRC Press.

Reza Masoodi, Krishna M Pillai, and Padma Prabodh Varanasi. 2008. Role of hydraulic and capillary radii in improving the effectiveness of capillary model in wicking. In ASME Summer Conference, facksonville, FL, USA.

Joe J Monaghan. 1994. Simulating free surface flows with SPH. Journal of computational physics 110, 2 (1994), 399-406.

Matthias Müller, David Charypar, and Markus Gross. 2003. Particle-based fluid simulation for interactive applications. In Proceedings of the 2003 ACM SIG GRAPH/Eurographics symposium on Computer animation. Eurographics Association, 154-159.

Michael B Nielsen and Ole Østerby. 2013. A two-continua approach to Eulerian simulation of water spray. ACM Transactions on Graphics (TOG) 32, 4 (2013), 67.

P Nithiarasu, KN Seetharamu, and T Sundararajan. 1997. Natural convective heat transfer in a fluid saturated variable porosity medium. International fournal of Heat and Mass Transfer 40, 16 (1997), 3955-3967.

Hilary Ockendon and John R Ockendon. 1995. Viscous flow. Vol. 13. Cambridge University Press.

Alexander Oron, Stephen H Davis, and S George Bankoff. 1997. Long-scale evolution of thin liquid films. Reviews of modern physics 69, 3 (1997), 931.

Oktar Ozgen, Marcelo Kallmann, Lynnette Es Ramirez, and Carlos Fm Coimbra. 2010. Underwater cloth simulation with fractional derivatives. ACM Transactions on Graphics (TOG) 29, 3 (2010), 23

Claudio Paniconi, Alvaro A Aldama, and Eric F Wood. 1991. Numerical evaluation of iterative and noniterative methods for the solution of the nonlinear Richards equation. Water Resources Research 27, 6 (1991), 1147-1163.

Saket Patkar and Parag Chaudhuri. 2013. Wetting of porous solids. IEEE transactions on visualization and computer graphics 19, 9 (2013), 1592-1604.

Amalendu Patnaik, RS Rengasamy, VK Kothari, and A Ghosh. 2006. Wetting and wicking in fibrous materials. Textile Progress 38, 1 (2006), 1-105.

Krishna M Pillai and Suresh G Advani. 1996. Wicking across a fiber-bank. fournal of colloid and interface science 183, 1 (1996), 100-110.

E Bruce Pitman and Long Le. 2005. A two-fluid model for avalanche and debris flows Philosophical Transactions of the Royal Society of London A: Mathematical, Physical and Engineering Sciences 363, 1832 (2005), 1573-1601.

Kumbakonam Ramamani Rajagopal and Lu Tao. 1995. Mechanics of mixtures. World Scientific.

Bo Ren, Chenfeng Li, Xiao Yan, Ming C Lin, Javier Bonet, and Shi-Min Hu. 2014. Multiplefluid SPH simulation using a mixture model. ACM Transactions on Graphics (TOG) 33, 5 (2014), 171.

Lorenzo Adolph Richards. 1931. Capillary conduction of liquids through porous mediums. Physics 1, 5 (1931), 318-333

Avi Robinson-Mosher, Tamar Shinar, Jon Gretarsson, Jonathan Su, and Ronald Fedkiw. 2008. Two-way Coupling of Fluids to Rigid and Deformable Solids and Shells. ACM Transactions on Graphics (TOG) 27, 3, Article 46 (Aug. 2008), 9 pages

Witawat Rungjiratananon, Zoltan Szego, Yoshihiro Kanamori, and Tomoyuki Nishita 2008. Real-time Animation of Sand-Water Interaction. Computer Graphics Forum 27,
7 (2008), 1887-1893.

Luc Scholtès, P-Y Hicher, François Nicot, Bruno Chareyre, and Félix Darve. 2009. On the capillary stress tensor in wet granular materials. International journal for numerical and analytical methods in geomechanics 33, 10 (2009), 1289-1313.

Aviv Segall, Orestis Vantzos, and Mirela Ben-Chen. 2016. Hele-shaw Flow Simulation with Interactive Control Using Complex Barycentric Coordinates. In Proceedings of the ACM SIGGRAPH/Eurographics Symposium on Computer Animation (SCA '16). Eurographics Association, Aire-la-Ville, Switzerland, Switzerland, 85-95.

MT Senoguz, FD Dungan, AM Sastry, and JT Klamo. 2001. Simulations and experiments on low-pressure permeation of fabrics: Part II-The variable gap model and prediction of permeability. Fournal of composite materials 35, 14 (2001), 1285-1322.

Toshihiro Shinohara, Jun-ya Takayama, Shinji Ohyama, and Akira Kobayashi. 2010 Extraction of yarn positional information from a three-dimensional CT image of textile fabric using yarn tracing with a filament model for structure analysis. Textile Research fournal 80, 7 (2010), 623-630.

Xiaoyu Song and Ronaldo I Borja. 2014. Mathematical framework for unsaturated flow in the finite deformation range. Internat. F. Numer. Methods Engrg. 97, 9 (2014), 658-682.

Alexey Stomakhin, Craig Schroeder, Chenfanfu Jiang, Lawrence Chai, Joseph Teran, and Andrew Selle. 2014. Augmented MPM for phase-change and varied materials. ACM Transactions on Graphics (TOG) 33, 4 (2014), 138.

Triantafyllos Stylianopoulos, Andrew Yeckel, Jeffrey J Derby, Xiao-Juan Luo, Mark S Shephard, Edward A Sander, and Victor H Barocas. 2008. Permeability calculations in three-dimensional isotropic and oriented fiber networks. Physics of Fluids 20, 12 (2008), 123601.

Deborah Sulsky, Zhen Chen, and Howard L Schreyer. 1994. A particle method for history-dependent materials. Computer methods in applied mechanics and engineering 118, 1-2 (1994), 179-196.

Andre Pradhana Tampubolon, Theodore Gast, Gergely Klár, Chuyuan Fu, Joseph Teran, Chenfanfu Jiang, and Ken Museth. 2017. Multi-species simulation of porous sand and water mixtures. ACM Transactions on Graphics (TOG) 36, 4 (2017), 105.

Karl von Terzaghi. 1923. Die berechnung der durchlassigkeitsziffer des tones aus dem verlauf der hydrodynamischen spannungserscheinungen. Sitzungsberichte der Akademie der Wissenschaften in Wien, Mathematisch-Naturwissenschaftliche Klasse, Abteilung IIa 132 (1923), 125-138.

Karl von Terzaghi. 1943. Theoretical soil mechanics. Vol. 18. Wiley Online Library

Bernhard Thomaszewski, Markus Wacker, Wolfgang Straßer, Etienne Lyard, C. Luible, Pascal Volino, M. Kasap, V. Muggeo, and Nadia Magnenat-Thalmann. 2007. Advanced Topics in Virtual Garment Simulation. In Eurographics 2007 - Tutorials, Karol Myszkowski and Vlastimil Havran (Eds.). The Eurographics Association.

Kiwon Um, Xiangyu Hu, and Nils Thuerey. 2017. Perceptual evaluation of liquid simulation methods. ACM Transactions on Graphics (TOG) 36, 4 (2017), 143.

Kiwon Um, Tae-Yong Kim, Youngdon Kwon, and JungHyun Han. 2013. Porous deformable shell simulation with surface water flow and saturation. Computer Animation and Virtual Worlds 24, 3-4 (2013), 247-254.

M Th Van Genuchten. 1980. A closed-form equation for predicting the hydraulic conductivity of unsaturated soils. Soil science society of America journal 44, 5 (1980), 892-898.

Orestis Vantzos, Omri Azencot, Max Wardeztky, Martin Rumpf, and Mirela Ben-Chen 2017. Functional Thin Films on Surfaces. IEEE transactions on visualization and computer graphics 23, 3 (2017), 1179-1192.

Huamin Wang, Gavin Miller, and Greg Turk. 2007. Solving general shallow wave equations on surfaces. In Proceedings of the 2007 ACM SIGGRAPH/Eurographics symposium on Computer animation. Eurographics Association, 229-238.

Yongxin Wang, Stephen Michielsen, and Hoon Joo Lee. 2013. Symmetric and asymmetric capillary bridges between a rough surface and a parallel surface. Langmuir 29, 35 (2013), 11028-11037.

Edward W Washburn. 1921. The dynamics of capillary flow. Physical review 17, 3 (1921), 273

JG Williams, CEM Morris, and BC Ennis. 1974. Liquid flow through aligned fiber beds. Polymer Engineering \& Science 14, 6 (1974), 413-419.

Reinhard Woltmann. 1792. Beiträge zur Hydraulischen Architectur. Vol. 2. Dieterich.

Xiao Yan, Yun-Tao Jiang, Chen-Feng Li, Ralph R Martin, and Shi-Min Hu. 2016. Multiphase sph simulation for interactive fluids and solids. ACM Transactions on Graphics (TOG) 35, 4 (2016), 79

Tao Yang, Jian Chang, Ming C Lin, Ralph R Martin, Jian J Zhang, and Shi-Min Hu. 2017. A unified particle system framework for multi-phase, multi-material visual simulations. ACM Transactions on Graphics (TOG) 36, 6 (2017), 224.

Tao Yang, Jian Chang, Bo Ren, Ming C Lin, Jian Jun Zhang, and Shi-Min Hu. 2015 Fast multiple-fluid simulation using Helmholtz free energy. ACM Transactions on Graphics (TOG) 34, 6 (2015), 201.

K Yazdchi and Stefan Luding. 2012. Towards unified drag laws for inertial flow through fibrous materials. Chemical engineering journal 207 (2012), 35-48.

Xinxin Zhang. 2015. A TBB Parallelized Liquid Solver Featuring Simple FLIP and AMGPCG Pressure Solver. https://github.com/zhxx1987/tbb_liquid_amgpcg. (2015). 